\title{
Regularized inversion of a two-dimensional integral equation with applications in borehole induction measurements
}

\author{
Orhan Arıkan ${ }^{1}$ \\ Schlumberger-Doll Research, Ridgefield, Connecticut
}

\begin{abstract}
Well bore measurements of conductivity, gravity, and surface measurements of magnetotelluric fields can be modeled as a two-dimensional integral equation with additive measurement noise. The governing integral equation has the form of convolution in the first dimension and projection in the second dimension. However, these two operations are not in separable form. In these applications, given a set of measurements, efficient and robust estimation of the underlying physical property is required. For this purpose, a regularized inversion algorithm for the governing integral equation is presented in this paper. Singular value decomposition of the measurement kernels is used to exploit convolution-projection structure of the integral equation, leading to a form where measurements are related to the physical property by a two-stage operation: projection followed by convolution. On the other hand, estimation of the physical property can be carried out by a two-stage inversion algorithm: deconvolution followed by back projection. A regularization method for the required multichannel deconvolution is given. Some important details of the algorithm are addressed in an application to wellbore induction measurements of conductivity.
\end{abstract}

\section{Introduction}

Integral equations have found wide applications in modeling physical phenomena. The most commonly used form is the Fredholm integral equation of the first kind, which has been extensively studied [Smithies, 1958; Delves and Mohamed, 1985; Hanson, 1971]. In this type of linear model, measurements are generally related to the underlying physical property as

$$
g(x)=\int K(x, y) f(y) d y+n(x),
$$

where $f$ is the physical property, $g$ is the measurement, and $n$ is the measurement noise which is assumed to be additive in this model. The kernel of this integral equation, $K$, models the underlying physical relationship between $f$ and $g$. In this paper the physical property is two-dimensional, and its relation to the measurement is governed by the following linear integral equation:

\footnotetext{
${ }^{1}$ Now at Electrical Engineering Department, Bilkent University, Ankara, Turkey.
}

Copyright 1994 by the American Geophysical Union.

Paper number 93RS02006.

0048-6604/94/93RS-02006\$08.00 $g_{i}(x)=\iint K_{i}\left(x-x^{\prime}, y\right) f\left(x^{\prime}, y\right) d y d x^{\prime}+n_{i}(x)$,

where a set of one-dimensional measurements $g_{i}(x)$, $1 \leq i \leq I$, are related to the two-dimensional physical property $f(x, y)$ through their respective kernel functions $K_{i}(x, y)$. The relationship is in the form of nonseparable projection in $y$ and a convolution in $x$. In oil exploration, this type of integral equation has been used in modeling wellbore measurements of conductivity and gravity of rock formations [Hunka et al., 1990; Sezginer, 1987]. These applications require a solution for $f(x, y)$ given a set of measurements $g_{i}(x)$. However, the inversion problem is highly ill-posed. In the absence of a prior model for $f(x, y)$, the inversion should provide an estimate $\hat{f}(x, y)$, which is in the observable space of measurements and robust to the measurement noise. For this purpose, an inversion algorithm which exploits the convolution-projection structure of (1) is given in this paper.

In the following section, singular function decompositions of measurement kernels are used to reformulate the measurement equation in the form of separable projection followed by convolution form. Subsequently, a multichannel deconvolution followed by backprojection algorithm for the inversion is given. Finally, as an example, an application to 
wellbore measurements of conductivity is presented showing some important details.

\section{An Inversion Algorithm}

The measurement relationship of (1) is used in many physical application areas including wellbore measurements of rock formations and profiling of magnetotelluric fields [Hunka et al., 1990; Sezginer, 1987; Torres-Verdin et al., 1990]. In these applications it is important to have an efficient algorithm for the estimation of $f(x, y)$ that is robust to the measurement noise. Often, there are some physical constraints on $f(x, y)$ such as positivity, monotonicity, etc. Some of these constraints can be incorporated into the estimation problem without significant increase in the complexity. In this section, an unconstrained estimation algorithm is proposed, and in section 3 a way of incorporating positivity constraint is shown.

The form of the measurement equation (1) is that of nonseparable convolution and projection operations. Singular function decomposition of the kernel $K_{i}(x, y)$ is useful in exploiting this structure of the measurement equation for an efficient estimation of the physical property. It is known that for $K_{i}$, whose energy is finite, there exists an approximation of $K_{i}$ in the following form:

$$
K_{i}(x, y) \approx \sum_{j=1}^{J_{i}} \lambda_{i j} u_{i j}(x) v_{i j}^{*}(y)
$$

with singular values $\lambda_{i 1} \geq \lambda_{i 2} \geq \cdots \geq \lambda_{i J_{i}}>0$, and singular functions satisfying

$$
\begin{aligned}
\lambda_{i j} u_{i j}(x) & =\int K_{i}(x, y) v_{i j}(y) d y \\
\lambda_{i j} v_{i j}(y) & =\int K_{i}^{*}(x, y) u_{i j}(x) d x \\
\delta_{j k} & =\int u_{i j}(x) u_{i k}^{*}(x) d x \\
\delta_{j k} & =\int v_{i j}(y) v_{i k}^{*}(y) d y .
\end{aligned}
$$

The error in this approximation decreases by increasing $J_{i}$. In the limit the error energy converges to zero. One way of obtaining such an expansion is by using matrix singular value decomposition
(SVD) of discretized $K_{i}$ [Chambers, 1976]. This provides an approximation to the required expansion. Another way of obtaining this decomposition is first forming left and right Hermitian kernels, $L H K_{i}(x, y)$ and $R H K_{i}(x, y)$, respectively, as

$$
\begin{aligned}
& \operatorname{LHK}_{i}(x, y)=\int K_{i}(x, z) K_{i}^{*}(y, z) d z \\
& \operatorname{RHK}_{i}(x, y)=\int K_{i}^{*}(z, x) K_{i}(z, y) d z
\end{aligned}
$$

and then by using the Hilbert-Schmidt theorem [Dunford and Schwartz, 1964; Keener, 1988; Pipkin, 1991], these two Hermitian kernels can be expanded into their singular function expansions,

$$
\begin{aligned}
& \operatorname{LHK}_{i}(x, y)=\sum_{j=1}^{\infty} \sigma_{i j} l_{i j}(x) l_{i j}^{*}(y) \\
& \operatorname{RHK}_{i}(x, y)=\sum_{j=1}^{\infty} \sigma_{i j} r_{i j}(x) r_{i j}^{*}(y),
\end{aligned}
$$

where the terms in these expansions are related to those in (3) as

$$
\begin{aligned}
u_{i j}(x) & =l_{i j}(x) \\
v_{i j}(x) & =r_{i j}(x) \\
\lambda_{i j} & =\sqrt{\sigma_{i j}} .
\end{aligned}
$$

In the next section, the decomposition of $K_{i}$ is obtained by discretization of the kernel and using SVD.

The measurement relation of (1) can be rewritten by replacing $K_{i}$ with its approximation in (2), giving

$$
\begin{aligned}
& g_{i}(x) \approx \iint\left\{\sum_{j=1}^{J_{i}} \lambda_{i j} u_{i j}\left(x-x^{\prime}\right) v_{i j}^{*}(y)\right\} f\left(x^{\prime}, y\right) d y d x^{\prime} \\
& +n_{i}(x) .
\end{aligned}
$$

In this form of the measurement relation, $g_{i}(x)$ is written as the weighted sum of convolutions of $u_{i j}(x)$ with projections of $f(x, y)$ onto $v_{i j}(y)$. Therefore the measurements are sensitive to those components of $f$ lying in the subspace generated by all $v_{i j}$. In the following discussion, this subspace is referred to as $y$-observable subspace. It is important 
to find an orthogonal basis to the $y$-observable subspace. Although $v_{i j}$ are orthogonal to each other when they have the same $i$ index as in (3), they may not be orthogonal to each other when they have different $i$ indices. Hence, in general, they do not form an orthogonal basis for the $y$-observable subspace for a more compact representation of the measurement relation. There are many practical ways of reconstructing an orthogonal basis for the $y$-observable subspace including Gram-Schmidt orthogonalization process applied to $v_{i j}$. Assuming such an orthogonalization procedure is performed over $v_{i j}$, providing an orthonormal basis $q_{m}$ for $1 \leq$ $m \leq M, v_{i j}$ values can be expressed as

$$
v_{i j}(y)=\sum_{m=1}^{M} \beta_{i j m} q_{m}(y) .
$$

Using this expression for $v_{i j}$ in (5), and changing orders of integrations and summations lead to the following approximation for the measurement relation:

$$
\begin{array}{r}
g_{i}(x)=\sum_{m=1}^{M}\left\{\int\left\{\sum_{j=1}^{J_{i}} \lambda_{i j} \beta_{i j m}^{*} u_{i j}\left(x-x^{\prime}\right)\right\}\right. \\
\left.\cdot\left\{\int q_{m}^{*}(y) f\left(x^{\prime}, y\right) d y\right\} d x^{\prime}\right\}+n_{i}(x) .
\end{array}
$$

The second summation in the above expression has all its terms independent of the unknown property $f(x, y)$; hence it can be precomputed as

$$
s_{i m}(x)=\sum_{j=1}^{J_{i}} \lambda_{i j} \beta_{i j m}^{*} u_{i j}(x) \text {. }
$$

For every $x^{\prime}$, the inner $y$-integral in (7) is the projection of $f\left(x^{\prime}, y\right)$ onto the basis component $q_{m}(y)$ of $y$-observable subspace. By defining this projection as

$$
p_{m}(x)=\int f(x, y) q_{m}^{*}(y) d y
$$

and using (8), the measurement relation can be rewritten in the following multichannel convolutional form:
$g_{i}(x)=\sum_{m=1}^{M}\left\{\int s_{i m}\left(x-x^{\prime}\right) p_{m}\left(x^{\prime}\right) d x^{\prime}\right\}+n_{i}(x)$.

This final form of the measurement relation suggests the following two-stage inversion algorithm: first estimate projections by performing multichannel deconvolution, and then backproject the resultant estimates for the estimate of the physical property

$$
\begin{gathered}
\hat{p}_{m}(x)=\sum_{i=1}^{I} \int h_{m i}\left(x-x^{\prime}\right) g_{i}\left(x^{\prime}\right) d x^{\prime} \\
\hat{f}(x, y)=\sum_{m=1}^{M} \hat{p}_{m}(x) q_{m}(y) .
\end{gathered}
$$

In these equations, $\hat{p}$ and $\hat{f}$ are used to denote the estimates for $p$ and $f$, respectively. Additive measurement noise should be taken into account in multichannel deconvolution stage. The choice of deconvolution filters $h_{m i}(x)$ depends on the available a priori information on the statistics of $p_{m}$ and the estimation criterion used. Usually, either no prior information or only first- and second-order statistics of $p_{m}$ are available. Depending on the available statistical description, maximum likelihood, least squares, or Bayesian estimation criteria can be used for this purpose.

In the Fourier transform domain, (11) can be written as

$$
\hat{P}_{m}(\omega)=\sum_{i=1}^{I} H_{m i}(\omega) G_{i}(\omega)
$$

where the upper-case functions are the Fourier transforms of the corresponding lower-case functions, or in vector-matrix notation

$$
\hat{\mathbf{P}}(\omega)=H(\omega) \mathbf{G}(\omega)
$$

with the use of boldface denoting a vector and the use of sans serif denoting a matrix. Similarly, (10) can be written in the Fourier transform domain as

$$
\mathbf{G}(\omega)=\mathbf{S}(\omega) \mathbf{P}(\omega)+\mathbf{N}(\omega),
$$

where $\mathbf{N}$ is the formal Fourier transform of the additive noise vector $\mathbf{n}$ which is assumed to be zero mean stationary vector process [Yaglom, 1962]. 
Hence the mean of $\mathbf{N}(\omega)$ is zero, and $\mathbf{N}\left(\omega_{1}\right)$ is independent of $\mathrm{N}\left(\omega_{2}\right)$ for $\omega_{1} \neq \omega_{2}$. Combining (14) and (15), we get

$$
\hat{\mathbf{P}}(\omega)=H(\omega) \mathbf{S}(\omega) \mathbf{P}(\omega)+H(\omega) \mathbf{N}(\omega) .
$$

The first and second terms in the right-hand side of this equation represents the signal and the noise contributions to the estimate, respectively. Some of the possible approaches are given below [Van Trees, 1968].

Maximum likelihood estimate. Assume no prior distribution on $\mathbf{P}(\omega)$ and Gaussian $\mathbf{N}(\omega)$ with autocorrelation $R_{N}(\omega)$. In order to obtain $\hat{\mathbf{P}}(\omega)$ that maximizes the probability of observing $\mathbf{G}(\omega), H(\omega)$ should be chosen as

$\mathrm{H}_{\mathrm{ML}}(\omega)=\left(\mathrm{S}^{H}(\omega) \mathrm{R}_{N}^{-1}(\omega) \mathrm{S}(\omega)\right)^{-1} \mathrm{~S}^{H}(\omega) \mathrm{R}_{N}^{-1}(\omega)$,

where a superscript $H$ denotes conjugate transpose operation. With the above assumption on the noise, this form also provides the optimal mean squared estimate.

Least squares estimate. Assume no prior distribution on $\mathbf{P}(\omega)$. In order to obtain $\hat{\mathbf{P}}(\omega)$ that minimizes $\|\mathbf{G}(\omega)-\mathbf{S}(\omega) \mathbf{P}(\omega)\|^{2}, H(\omega)$ should be chosen as

$$
\mathrm{H}_{\mathrm{LS}}(\omega)=\left(\mathbf{S}^{H}(\omega) \mathrm{S}(\omega)\right)^{-1} \mathrm{~S}^{H}(\omega)
$$

Maximum a posteriori estimate. Assume distribution of both $\mathbf{P}(\omega)$ and $\mathbf{N}(\omega)$ are independent Gaussian with autocorrelations $R_{P}(\omega)$ and $R_{N}(\omega)$, respectively. In order to obtain $\hat{\mathbf{P}}(\omega)$, which is the most probable $\mathbf{P}(\omega)$ after observing $\mathbf{G}(\omega), H(\omega)$ should be chosen as

$$
\begin{gathered}
\mathrm{H}_{\mathrm{MAP}}(\omega)=\left(\mathrm{S}^{H}(\omega) \mathrm{R}_{N}^{-1}(\omega) \mathrm{S}(\omega)\right. \\
\left.+\mathrm{R}_{P}^{-1}(\omega)\right)^{-1} \mathrm{~S}^{H}(\omega) \mathrm{R}_{N}^{-1}(\omega)
\end{gathered}
$$

The above listed forms for $H(\omega)$ do not depend on the Fourier transform of the current measurement. They all can be precomputed at distinct frequencies and then can be inverse Fourier transformed to obtain the set of filters for $h_{m i}(x)$. This helps reduce the computational complexity of the algorithm when it is used on many different measurement sets.

In the absence of a priori information on the statistics of $\mathbf{P}(\omega)$, one can use $\mathrm{H}_{\mathrm{ML}}(\omega)$, as given above with the corresponding error covariance:

$E\left\{\left(\mathbf{P}(\omega)-H_{M L}(\omega) \mathbf{G}(\omega)\right)\left(\mathbf{P}(\omega)-H_{M L}(\omega) \mathbf{G}(\omega)\right)^{H}\right\}$

$$
=\left(\mathrm{S}^{H}(\omega) \mathrm{R}_{N}^{-1}(\omega) \mathrm{S}(\omega)\right)^{-1} .
$$

Although the mean of the maximum likelihood estimate $\hat{\mathbf{P}}_{\mathrm{ML}}(\omega)$ is the true $\mathbf{P}(\omega)$, its covariance given in (20) may be unacceptably large. This is likely to happen at those frequencies where $S(\omega)$ has a relatively small singular value. Therefore in literature, when this technique is used, the matrix inversion is usually regularized as

$$
\begin{gathered}
\mathrm{H}_{\mathrm{MLR}}(\omega)=\left(\mathrm{S}^{H}(\omega) \mathrm{R}_{N}^{-1}(\omega) \mathrm{S}(\omega)\right. \\
\left.+\mu^{2}(\omega) \mathrm{l}\right)^{-1} \mathrm{~S}^{H}(\omega) \mathrm{R}_{N}^{-1}(\omega),
\end{gathered}
$$

where $\mu(\omega)$ is the regularization parameter which can be a fixed function of $\omega$ or can be chosen adaptively after acquiring the measurements [Tikhonov and Arsenin, 1977; Dennis and Schnabel, 1983]. However, both of these approaches have important drawbacks. Since the latter approach depends on the current measurements, it requires significant amount of computation to be performed to find the deconvolution filter set. Although the first approach does not require a change in the filter set every time this algorithm is used, in practice it is difficult to find a satisfactory regularization parameter when there are many sampling frequencies used and the noise at the chosen frequencies are not identically distributed. In this paper, a different approach is taken for the computation of $H(\omega)$. In this approach, $H(\omega)$ is going to be chosen as the solution to the following optimization problem: minimize

$$
\|\mathrm{H}(\omega) \mathrm{S}(\omega)-\|_{F}^{2}
$$

subject to trace

$$
\left(\mathrm{H}(\omega) \mathrm{R}_{N}(\omega) \mathrm{H}(\omega)^{H}\right) \leq \varepsilon^{2}(\omega),
$$

where \|\|$_{F}$ denotes the Frobenius matrix norm which is defined to be the square root of the sum of magnitude squares of all the matrix entries [Golub and Van Loan, 1989]. This choice for $H(\omega)$ and $H_{\varepsilon}(\omega)$ can be motivated by observing that when the cost in (22) is small, the mean of the estimates gets close to the true values, and the constraint puts an upper bound, $\varepsilon^{2}(\omega)$, on the expected energy of the noise around this mean. An iterative optimization algorithm for the solution of this optimization problem is given in the appendix. This approach has been found to be the most appropriate one for the application given in the next section. As it is shown 
in the appendix, $H_{\varepsilon}(\omega)$ is the same form given in (21):

$\mathrm{H}_{\varepsilon}(\omega)=\left(\mathrm{S}^{H}(\omega) \mathrm{R}_{N}^{-1}(\omega) \mathrm{S}(\omega)+\mu^{2} \mathrm{I}\right)^{-1} \mathrm{~S}^{H}(\omega) \mathrm{R}_{N}^{-1}(\omega)$,

with a specific choice of $\mu$ that solves the following equation:

$$
\sum_{i=1}^{M}\left(\frac{\eta_{i}}{\eta_{i}^{2}+\mu^{2}}\right)^{2}=\varepsilon(\omega)^{2},
$$

where $\eta_{i}$ are the singular values of $\mathrm{R}_{N}(\omega)^{-1 / 2} \mathrm{~S}(\omega)$. Therefore this approach provides a way of optimally choosing the regularization parameter $\mu$, given the maximum allowed noise energy in the estimate.

\section{An Application in Well Logging}

In this section the proposed inversion algorithm is applied to induction measurements of conductivity in well logging for oil exploration. Also, some important details of the algorithm are examined.

In oil exploration, various types of measurements are used to acquire properties of rock formations and their fluid contents in an area of interest. Some of these measurements are obtained at the surface of Earth, such as surface geological and seismic studies, and some are acquired in and between wells drilled into the Earth [Ellis, 1987]. One of these measurements performed in a single wellbore is the induction measurement of conductivity to separate resistive hydrocarbon zones from usually less resistive water bearing zones. The geometry of data acquisition is shown in Figure 1. In this figure a measurement device with one transmitting and two receiving coils, all centered at the borehole axis, is shown. To prevent possible blowouts, a mixture of chemicals and fluids, called the drilling mud, is constantly pumped into the well at a pressure higher than the pressure of the fluid or gas in rock formation. This results in an invasion of the rock formation with mostly the fluid component of the mud and an accumulation of the solid component on the borehole wall. Although this invasion process changes the original conductivity distribution around the borehole significantly, the extent and actual shape of the invaded region is rather difficult to predict. The main goal of induction measurements is to estimate the conductivity distribution beyond this invaded zone. For this purpose,

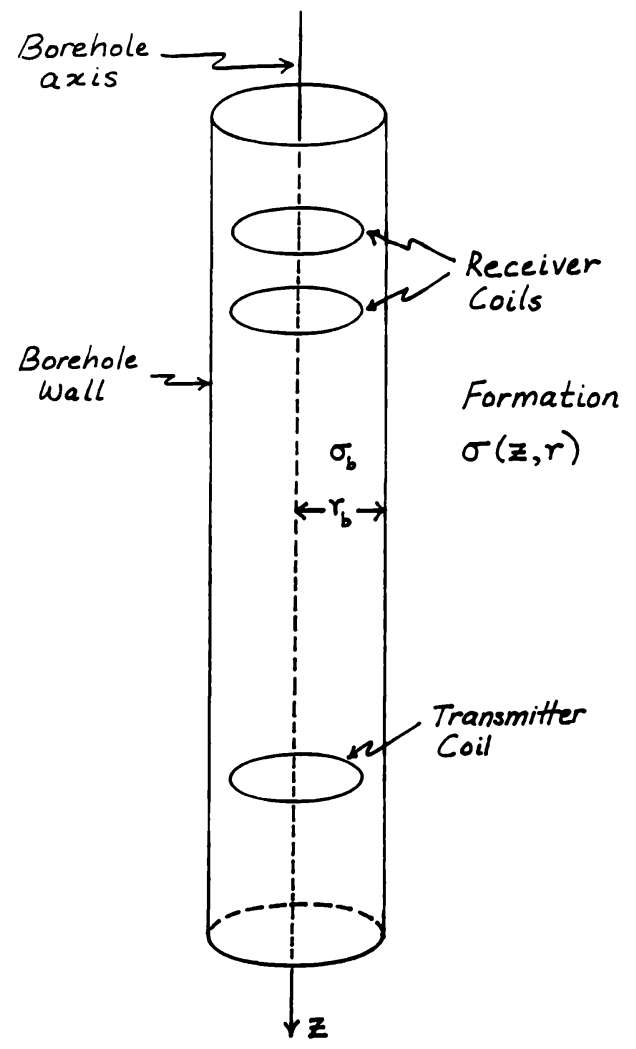

Figure 1. Geometry of data acquisition in wellbore induction measurements: the conductivity in cylindrical borehole is constant $\sigma_{b}$, and formation conductivity is function of $z$ and $r$ only.

an array induction measurement tool with 14 subarrays has been developed [Barber and Rosthal, 1991]. Each subarray of this tool consists of one transmitter and two receiver coils, similar to the one shown in Figure 1, with main coil spacings ranging from a few centimeters to almost $2 \mathrm{~m}$. The transmitter coil, excited by alternating currents, induces eddy currents following circular paths along the borehole. These eddy currents create a secondary magnetic field which induces currents in the receiver coils [Moran and Kunz, 1962]. Each subarray measurement is formed by a combination of these received currents giving inphase and quadrature channels. In total there are 28 real measurements, 14 inphase and 14 quadrature. The dependence of measurements to the formation conductivity is nonlinear. However, by using the Born approximation, measurement relation to physical properties can be linearized around a background conductivity distribution, which is usually chosen 
as a constant [Moran and Kunz, 1962]. For a background conductivity of $\sigma_{0}$, this approximate measurement relation can be written in cylindrical coordinates as

$$
\delta l_{i}(z)=\int_{-\infty}^{\infty} \int_{0}^{\infty} K_{i}\left(z-z^{\prime}, r, \sigma_{0}\right) \delta \sigma\left(z^{\prime}, r\right) d r d z^{\prime}+n_{i}(z)
$$

where $\delta l_{i}(z)$ is the deviation of measured current from that dictated by the background medium, $\delta \sigma(z, r)$ is the difference between actual conductivity distribution $\sigma(z, r)$ and $\sigma_{0}$, and $K_{i}\left(z, r, \sigma_{0}\right)$ is the Born kernel which depends on the actual configuration of the $i$ th array as well as $\sigma_{0}$. The validity of the linearized measurement equation is limited to the small scatterer case and has been studied in detail in the literature [Habashy and Mittra, 1987; Habashy et al., 1993]. It has been found useful to use the following approximations for a better modeling of the measurements. First, the conductivity distribution $\sigma(z, r)>0$ can be written as

$$
\sigma(z, r)=e^{\psi(z, r)},
$$

which leads to the following approximation of $\delta \sigma(z, r)$

$$
\begin{aligned}
\delta \sigma(z, r) & =\sigma(z, r)-\sigma_{0} \\
& =e^{\psi(z, r)}-e^{\psi_{0}} \\
& =e^{\psi_{0}}\left(e^{\delta \psi(z, r)}-1\right) \\
& \approx \sigma_{0} \delta \psi(z, r),
\end{aligned}
$$

where $\delta \psi(z, r)=\psi(z, r)-\psi_{0}$ and in the last step, $\delta \psi(z, r)$ assumed to be small. Also, $\delta l_{i}(z)$ can be approximated as

$$
\delta l_{i}(z) \approx l_{i 0}\left(\log \left(l_{i}(z)\right)-\log \left(l_{i 0}\right)\right)
$$

in the following steps

$$
\begin{aligned}
\log \left(l_{i}(z)\right)-\log \left(l_{i 0}\right) & =\log \left(\frac{l_{i}(z)}{l_{i 0}}\right) \\
& =\log \left(\frac{l_{i 0}+\delta l_{i}(z)}{l_{i 0}}\right) \\
& =\log \left(1+\frac{\delta l_{i}(z)}{l_{i 0}}\right)
\end{aligned}
$$

$$
\approx \frac{\delta l_{i}(z)}{l_{i 0}}
$$

where the last ratio is assumed to be small. By using these two approximations, the measurement equation can be rewritten in the following form:

$$
\begin{aligned}
l_{i 0}\left(\log \left(l_{i}(z)\right)-\log \left(l_{i 0}\right)\right) \\
\quad=\int_{-\infty}^{\infty} \int_{0}^{\infty} K_{i}\left(z-z^{\prime}, r, \sigma_{0}\right) \sigma_{0} \delta \psi\left(z^{\prime}, r\right) d r d z^{\prime}+n_{i}(z) .
\end{aligned}
$$

This expression is in the form of (1), where $l_{i 0}(\log$ $\left.\left(l_{i}(z)\right)-\log \left(l_{i 0}\right)\right), K_{i}\left(z, r, \sigma_{0}\right)$, and $\sigma_{0} \delta \psi(z, r)$ are measurement, kernel, and physical property functions, respectively. For the sake of simplicity, in the remainder of this section, measurement relation of (1) is used instead of (27). In general, inphase measurement kernels have more energy than their corresponding quadrature measurement kernels. In Figure $2, K_{1}(z, r)$ and $K_{15}(z, r)$, inphase and quadrature kernels of the shortest subarray, and in Figure $3, K_{14}(z, r)$ and $K_{28}(z, r)$, inphase and quadrature kernels of the longest subarray, are shown. Since in this application both the measurements and the unknown property are in the same units of conductivity, Siemens per meter, the measurement kernels and all quantities, such as singular values and the functions derived from them, are unitless. As seen from these two figures, shorter subarrays have more of their energy closer to the borehole axis, and the kernels for the inphase measurements are both sharper and shallower than the quadrature channels. However, one of the most important features of these figures is that the energy of the kernels decreases as $r$, the distance away from the borehole axis, increases. This results in insensitivity to the features of conductivity beyond some distance away from the borehole axis. Therefore the measurement equation can be further approximated as

$g_{i}(z)=\int_{-\infty}^{\infty} \int_{0}^{r_{1}} K_{i}\left(z-z^{\prime}, r\right) f\left(z^{\prime}, r\right) d r d z^{\prime}+n_{i}(z)$

for $r_{1}$ large enough to capture most of the energy of the individual kernels. In this and other applications mentioned earlier, it is observed that fast decay of the measurement kernels results in observable $r$ subspace basis components, $q_{i}(r)$, in which the 

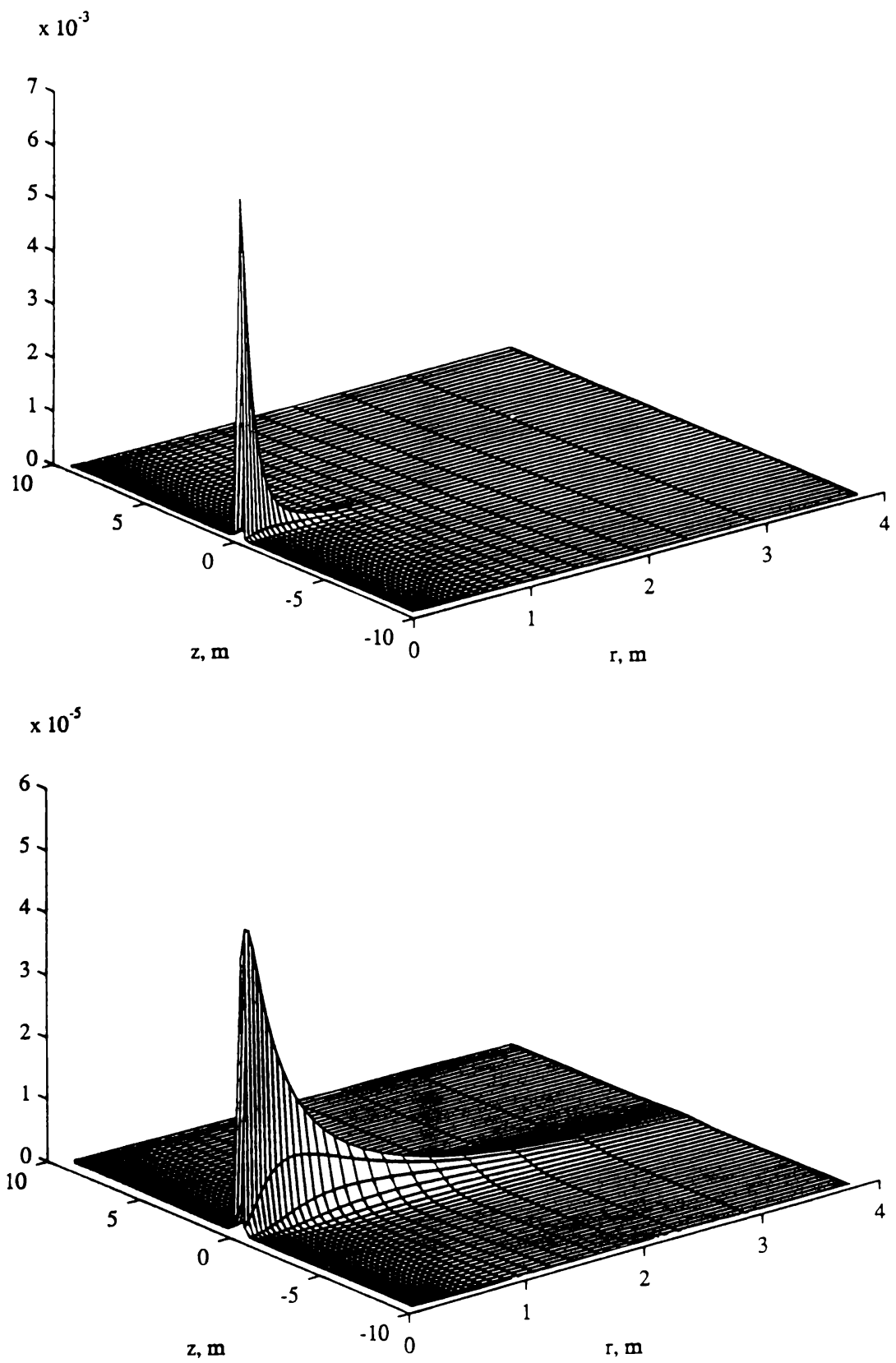

Figure 2. Inphase and quadrature measurement kernels of the shortest subarray.

dominant first few has a similar fast decay property. Since regularized inversion algorithms provide a reconstruction based on these first few resolvable components, the deeper features in the conductivity distribution cannot be accurately recovered. To overcome this problem as much as possible, a variable change in the $r$ integral is considered here. Changing $r$ variable with $r^{\prime}$, such that $r=\phi\left(r^{\prime}\right)$ with differentiable $\phi$, the measurement equation can be rewritten as

$$
g_{i}(z)=\int_{-\infty}^{\infty} \int_{r_{0}^{\prime}}^{r_{i}^{\prime}} K_{i}\left(z-z^{\prime}, \phi\left(r^{\prime}\right)\right) \frac{d \phi\left(r^{\prime}\right)}{d r^{\prime}}
$$

$$
\cdot f\left(z^{\prime}, \phi\left(r^{\prime}\right)\right) d r^{\prime} d z^{\prime}+n_{i}(z)
$$



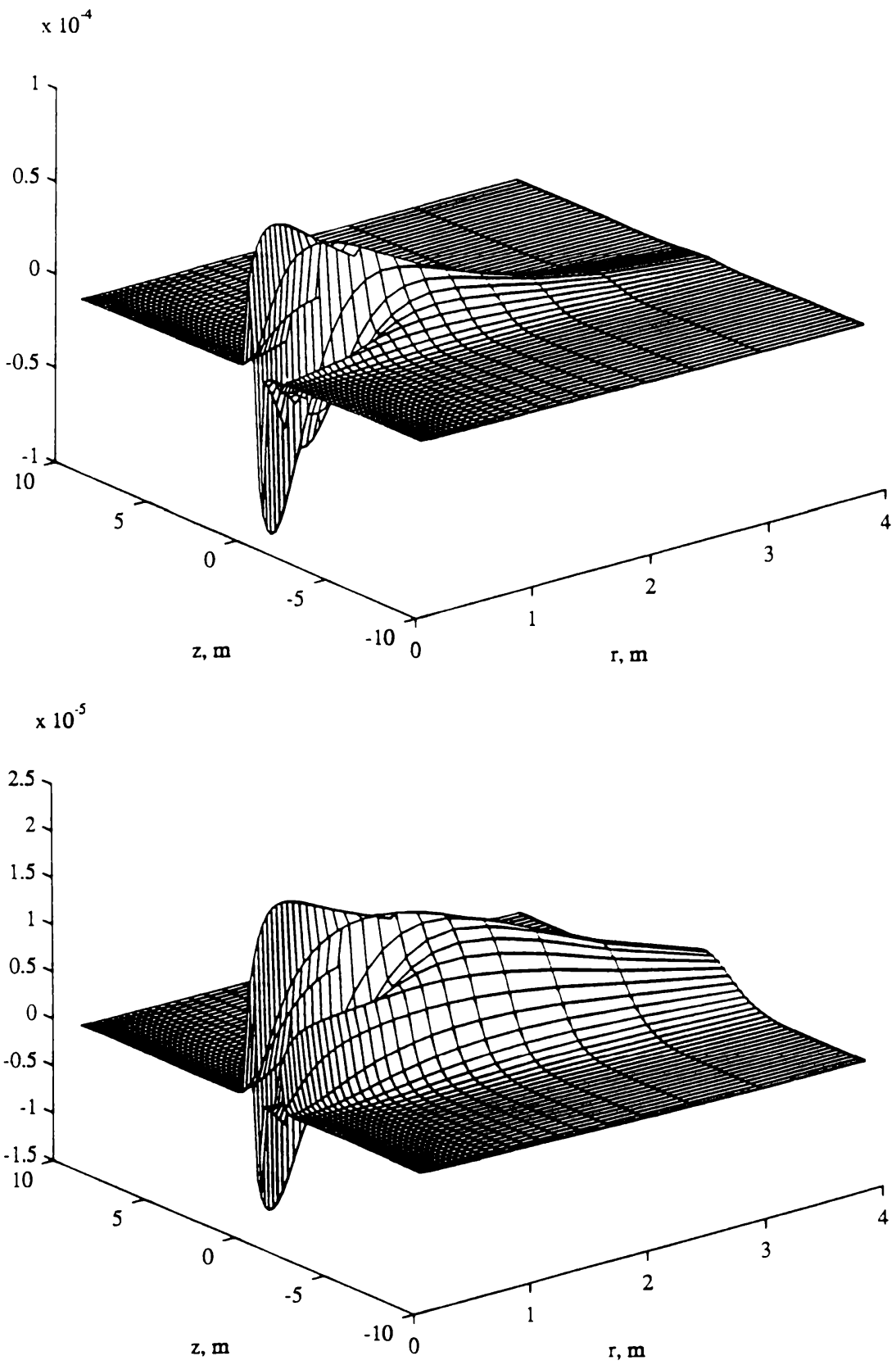

Figure 3. Inphase and quadrature measurement kernels of the longest subarray.

$g_{i}(z)=\int_{-\infty}^{\infty} \int_{r_{0}^{\prime}}^{r_{1}^{\prime}} \bar{K}_{i}\left(z-z^{\prime}, r^{\prime}\right) \bar{f}\left(z^{\prime}, r^{\prime}\right) d r^{\prime} d z^{\prime}+n_{i}(z)$,

where

$$
\bar{K}_{i}\left(z, r^{\prime}\right)=K_{i}\left(z, \phi\left(r^{\prime}\right)\right) \frac{d \phi\left(r^{\prime}\right)}{d r^{\prime}}
$$

and

$$
\bar{f}\left(z, r^{\prime}\right)=f\left(z, \phi\left(r^{\prime}\right)\right) .
$$

In general, the new measurement kernel in (31) has different basis components $\bar{q}_{i}\left(r^{\prime}\right)$ than the original kernel. In this application a monotonic increasing $\phi$ function (Figure 4) has been chosen so that in the 


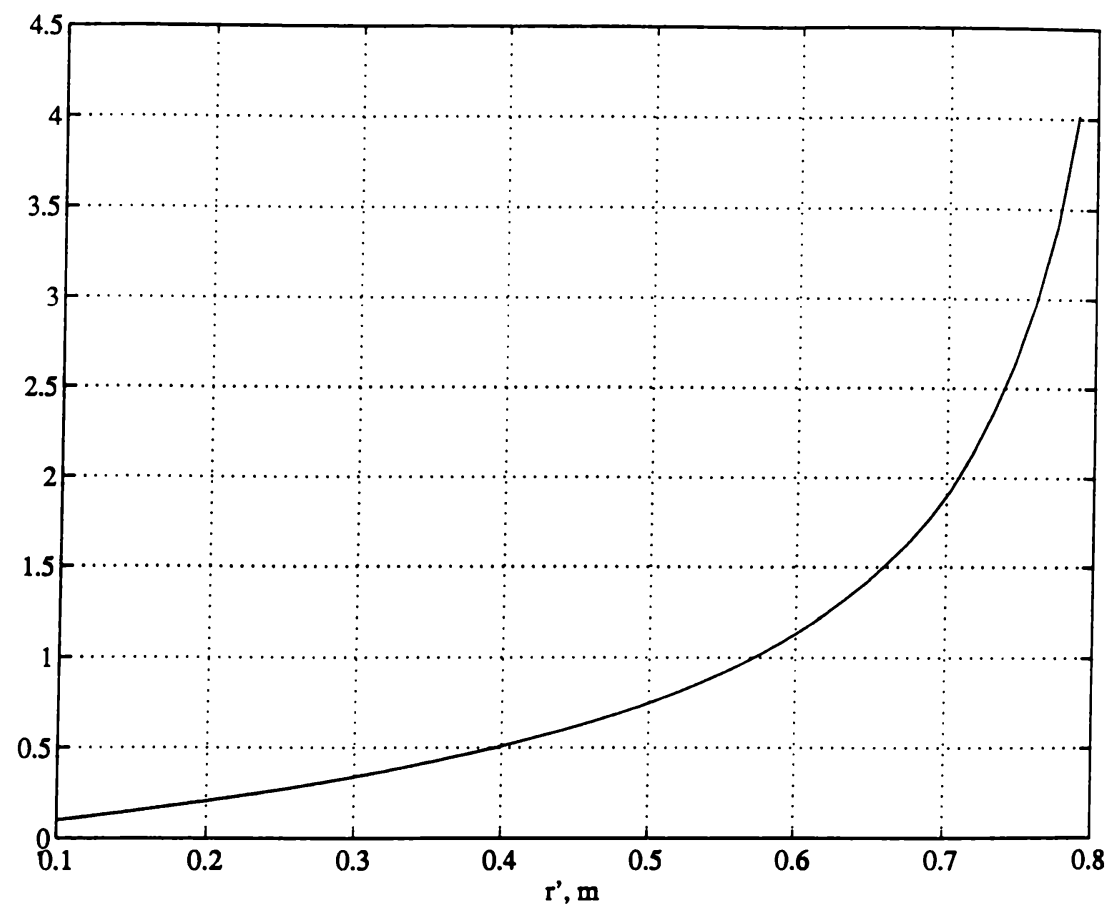

Figure 4. Monotonic increasing $\phi\left(r^{\prime}\right)$ used in change of radial integral variable.

new $r^{\prime}$ coordinate system, the cylindrical shells of conductors with different radii have approximately the same total energy contribution to the measurements. The chosen $\phi\left(r^{\prime}\right)$ is a piecewise defined function with three regions. Every piece of $\phi\left(r^{\prime}\right)$ is an exponential function. However, it is important to note that different applications may require different forms for this function.

Figures 5 and 6 show the first nine dominant components of observable $r$ and $r^{\prime}$ subspaces corresponding to kernels $K_{i}(z, r)$ and $\bar{K}_{i}\left(z, r^{\prime}\right)$ for a background conductivity of $0.5 \mathrm{~S} \mathrm{~m}^{-1}$. These components have been computed by using $\mathrm{QR}$ decomposition of matrices obtained by sampling measurement kernels, $K_{i}(n \Delta z, m \Delta r)$ and $\bar{K}_{i}\left(n \Delta z, m \Delta r^{\prime}\right)$, with $256 z, 50 r$, and $50 r^{\prime}$ samples. These figures show that $\bar{q}_{i}\left(r^{\prime}\right)$ have significantly more energy deeper into the formation, making it possible to recover the deeper features of conductivity variation. After this important digression, in the rest of this section, the inversion algorithm is developed as outlined in the previous section based on (30).

Singular value decomposition of the matrices with dimension $256 \times 50$, obtained by discretizing the kernels, $\overline{\mathrm{K}}_{i}=\bar{K}_{i}\left(n \Delta z, m \Delta r^{\prime}\right)$, provides left and right singular vectors as well as the singular values as

$$
\overline{\mathrm{K}}_{i}=\sum_{j=1}^{50} \lambda_{i j} \mathbf{u}_{i j} \mathbf{v}_{i j}^{H},
$$

which is in parallel structure to (2). As seen in Figure 7, except from the first few, most of the singular values of each kernel is very small. At this point, only those singular values, which are greater than $1 \%$ of the maximum of all the singular values are kept, resulting

$$
\overline{\mathrm{K}}_{i} \approx \sum_{j=1}^{J_{i}} \lambda_{i j} \mathbf{u}_{i j} \mathbf{v}_{i j}^{H},
$$

where $J_{i}$ is the number of singular values greater than the set threshold. The choice of the threshold depends on the particular application; however, the number of measurement channels and the noise level of each measurement should be taken into account in this decision. For the computation of the orthonormal basis component, $\bar{q}_{m}, \mathrm{QR}$ decomposi- 


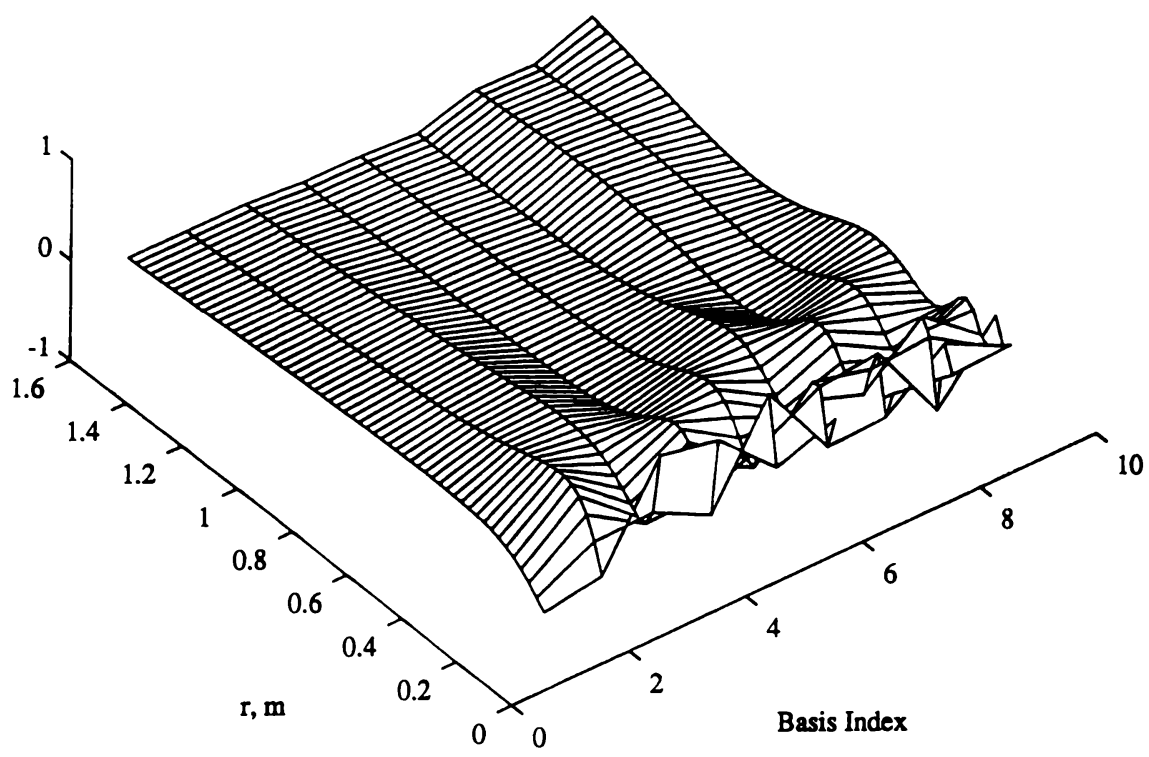

Figure 5. First nine components of observable radial subspace corresponding to original $K_{i}(z, r)$ kernels.

tion of the matrix whose columns are the right singular functions is used, giving

$$
\left[v_{11} \cdots v_{1 J_{1}}: \cdots: v_{281} \cdots v_{28} J_{28}\right]=\mathrm{QR},
$$

where $Q$ is a unitary matrix and $R$ is an upper triangular matrix [Golub and Van Loan, 1989]. As shown by the contour plot of $R$ given in Figure 8, effective rank of the matrix on the left-hand side of (35) is very small. By using SVD for this matrix and the known noise statistics of the measurements, the effective rank is found to be 11 . Thus only the first 11 columns of the $Q, \overline{\mathbf{q}}_{m}, 1 \leq m \leq 11$, form the required basis for the observable subspace, and $v_{i j}$ can be expressed in terms of

$$
v_{i j}=\sum_{m=1}^{11} \beta_{i j m} \bar{q}_{m}
$$

similar to (6). The following discretized form of (8) can be used for the computation of $s_{i m}$ :

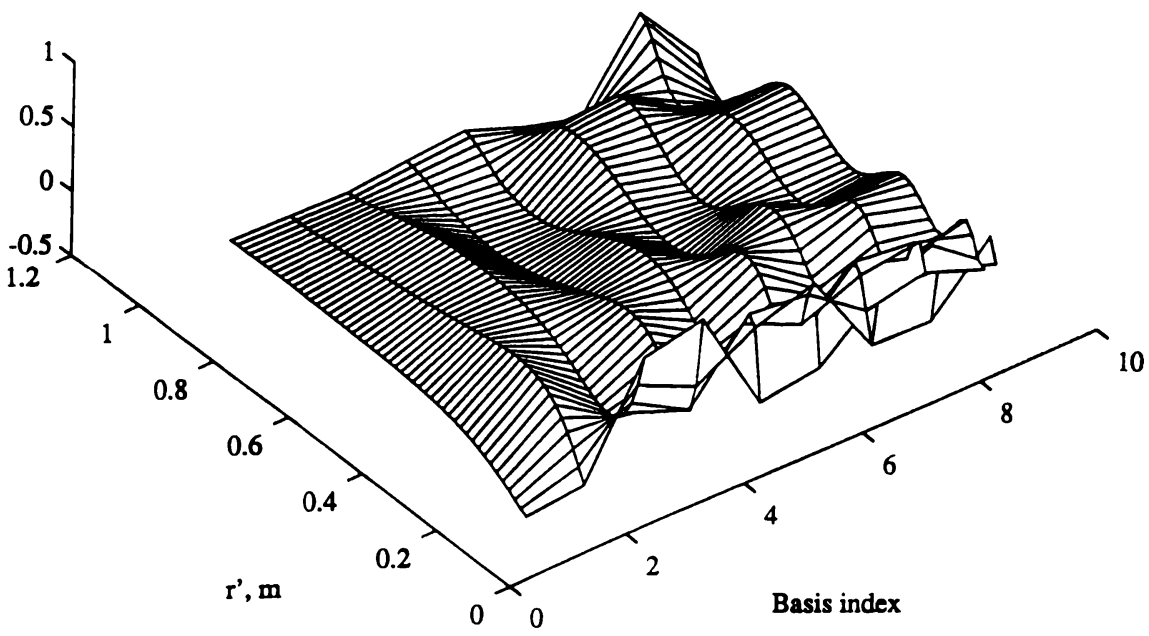

Figure 6. First nine components of observable radial subspace corresponding to new $\bar{K}_{i}\left(z, r^{\prime}\right)$ kernels. 


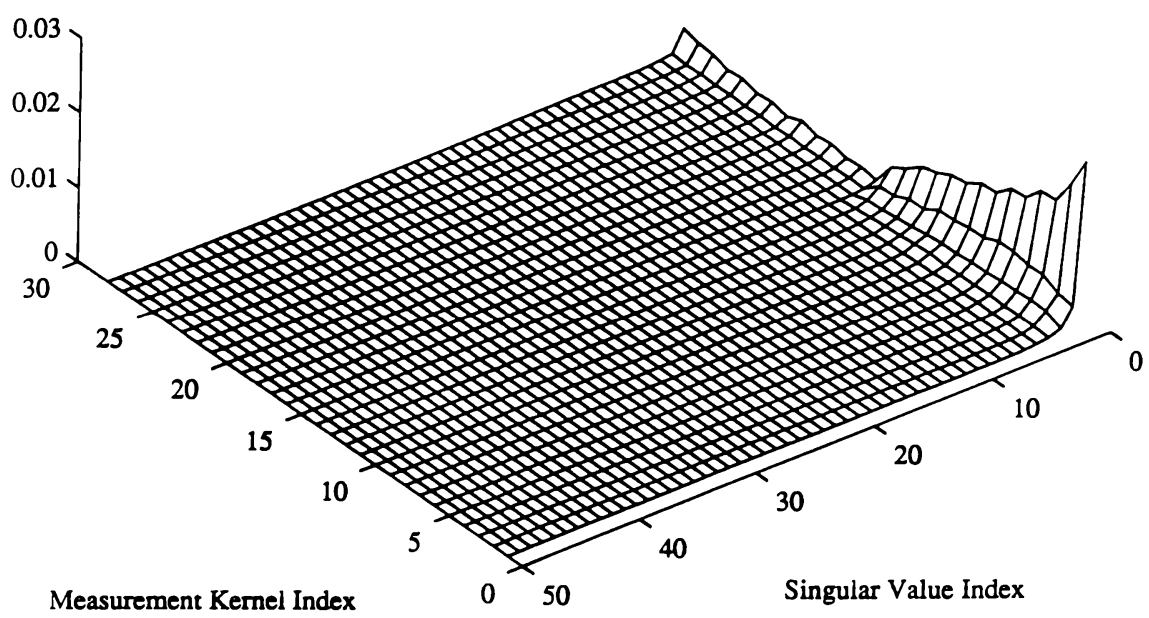

Figure 7. Singular values of 28 measurement kernels: kernel indexing is such that first 14 correspond to inphase kernels from the shortest to longest, and second 14 correspond to their respective quadrature kernels.

$$
\mathbf{s}_{i m}=\sum_{j=1}^{J_{i}} \lambda_{i j} \beta_{i j m}^{*} u_{i j}
$$

Figures 9 and 10 show as three-dimensional mesh plots two matrices whose columns are $\mathbf{s}_{1 m}$ and $\mathbf{s}_{14 m}$, respectively. The first of these matrices corresponds to the real part of the measurements obtained by the shortest array, and the second one corresponds to that obtained by the longest array. As its seen from the comparison of Figures 9 and 10 , shorter arrays have more localized $s_{i m}$. In general, $\mathbf{s}_{i m}$ has lower energy for larger $m$, making it

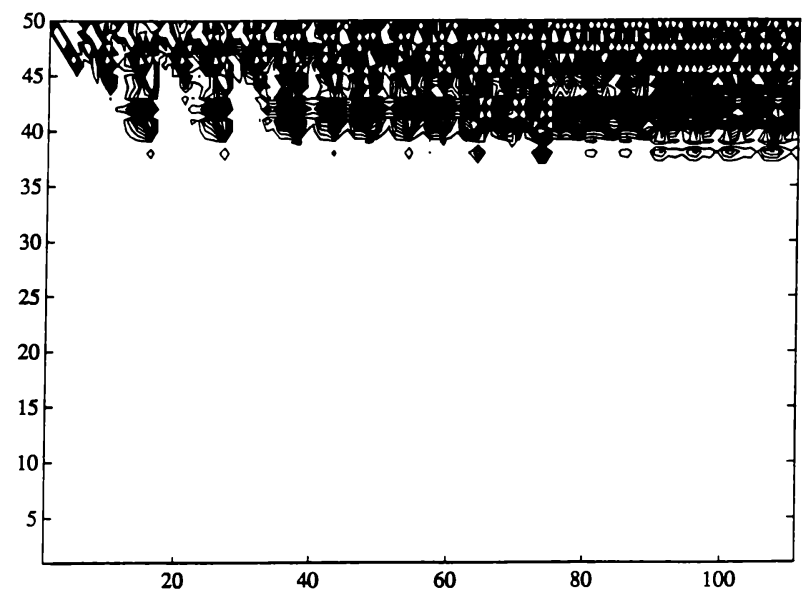

Figure 8. Contour plot of $R$ with 100 equally spaced contour levels showing that only the first few rows have significant energy. more difficult to estimate the corresponding projection $\mathbf{p}_{m}$ in the presence of measurement noise.

In the absence of reliable a priori information about projections, it has been found that the deconvolution filters obtained in the frequency domain by optimizing (22) under the constraint given in (23) provides better results than either maximum likelihood, (17) or least squares estimates, (18). This optimization is performed at 256 uniformly spaced spatial frequency samples, $\omega_{k}=2 \pi k /(256 \Delta z),-128$ $\leq k \leq 127$. Additive noise of each measurement has been experimentally determined to be uncorrelated zero mean stationary white process with different variances. In this application, $\varepsilon\left(\omega_{k}\right)$ is chosen as constant $\varepsilon_{0}$ for $|k| \leq k_{0}$ and zero outside this interval. Two important observations should be made at this point. Estimates with higher-frequency content can be obtained by increasing $k_{0}$; however, this also increases the variance of the estimates. Also, by increasing $\varepsilon_{0}$, the mean of the estimates can be made closer to the actual values; however, this causes an increase in the variance of the estimates. After finding optimal $\mathrm{H}^{*}\left(\omega_{k}\right)$ as explained in the appendix, the spatial domain deconvolution filters are found by inverse Fourier transform, as

$h_{i m}(n \Delta z)$

$$
=\frac{1}{N \Delta z} \sum_{k=-k_{0}}^{k_{0}} H_{i m}^{*}\left(\omega_{k}\right) \text { wndw }(k) \exp \left\{\frac{2 \pi}{256} k n\right\},
$$




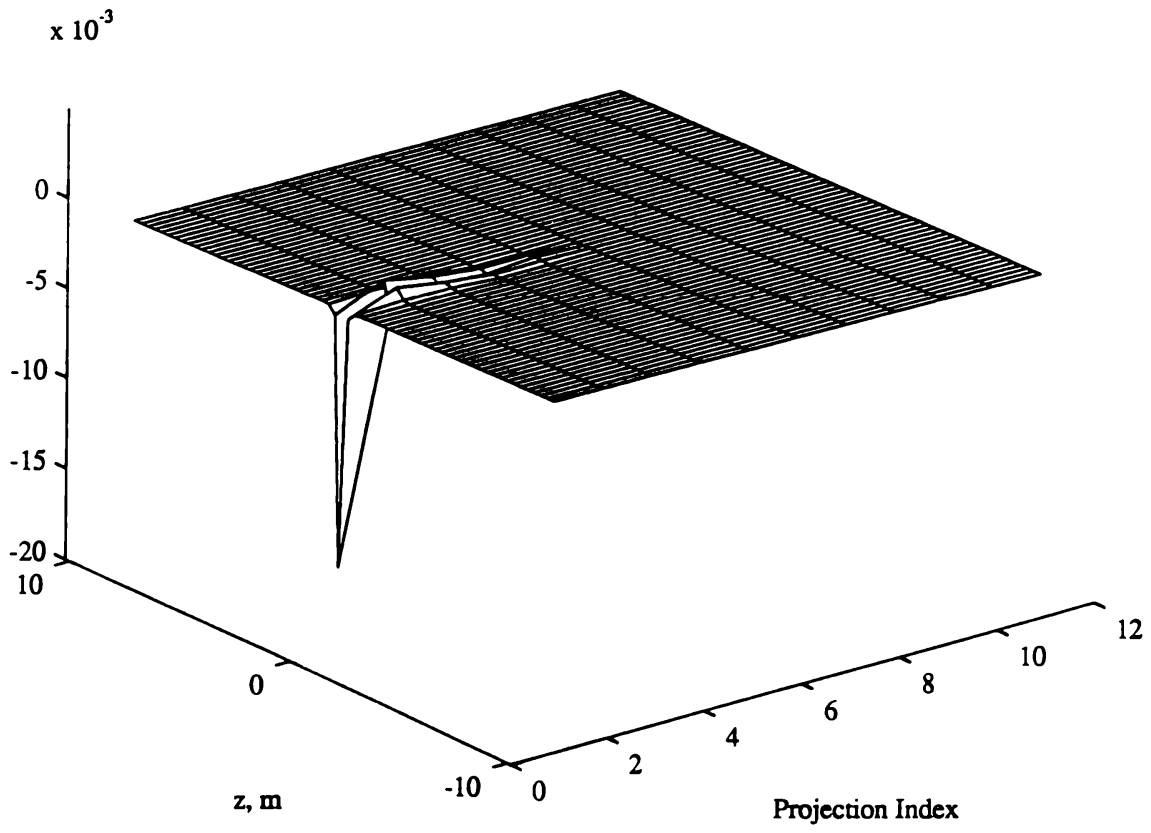

Figure 9. The vector $\mathbf{s}_{1 m}$ : filters that relate projections $\mathbf{p}_{m}, 1 \leq m \leq 11$, to the inphase measurements of the shortest subarray.

where $\operatorname{wndw}(k)$ is a window function which can be used to reduce highly oscillatory deconvolution filters resulting from Gibbs phenomenon due to abrupt frequency domain transitions. There are many available window functions such as Hamming, Kaiser, and Blackman that can be used here. Usually, the application in hand determines which one of them should be used. In this particular

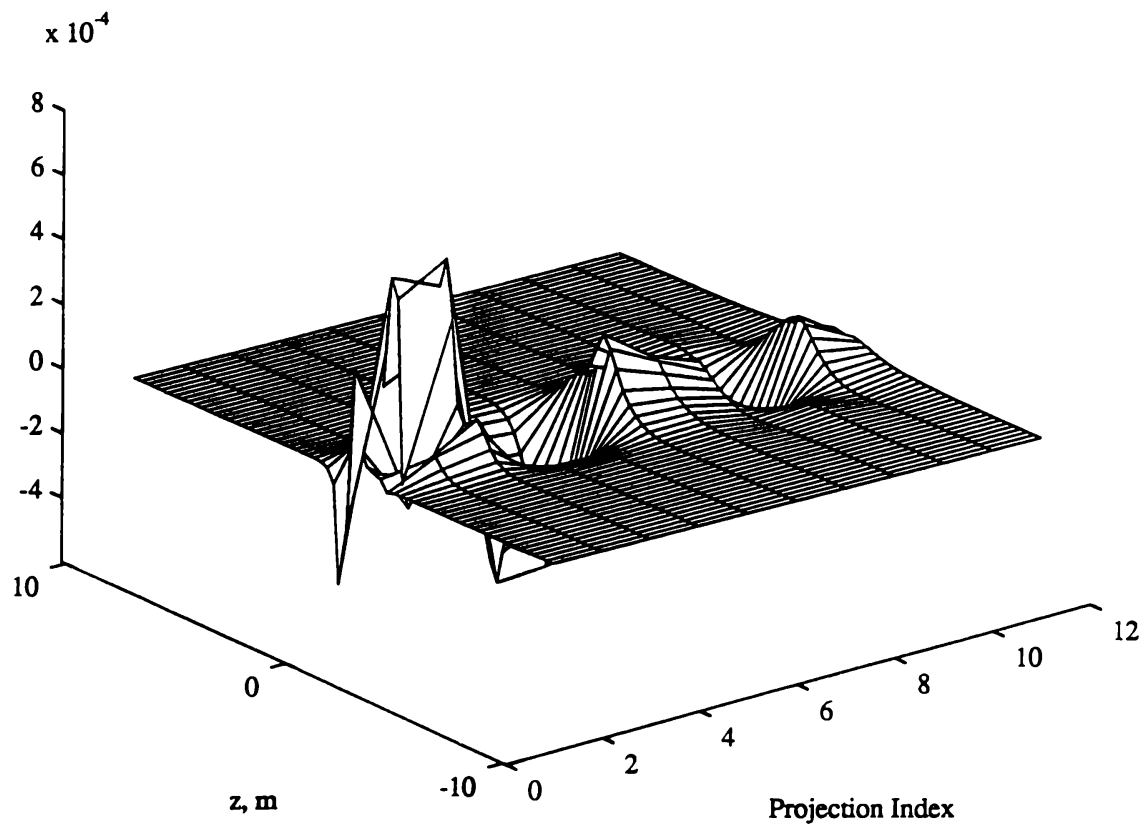

Figure 10. The vector $s_{14 m}$ : filters that relate projections $\mathbf{p}_{m}, 1 \leq m \leq 11$, to the inphase measurements of the longest subarray. 


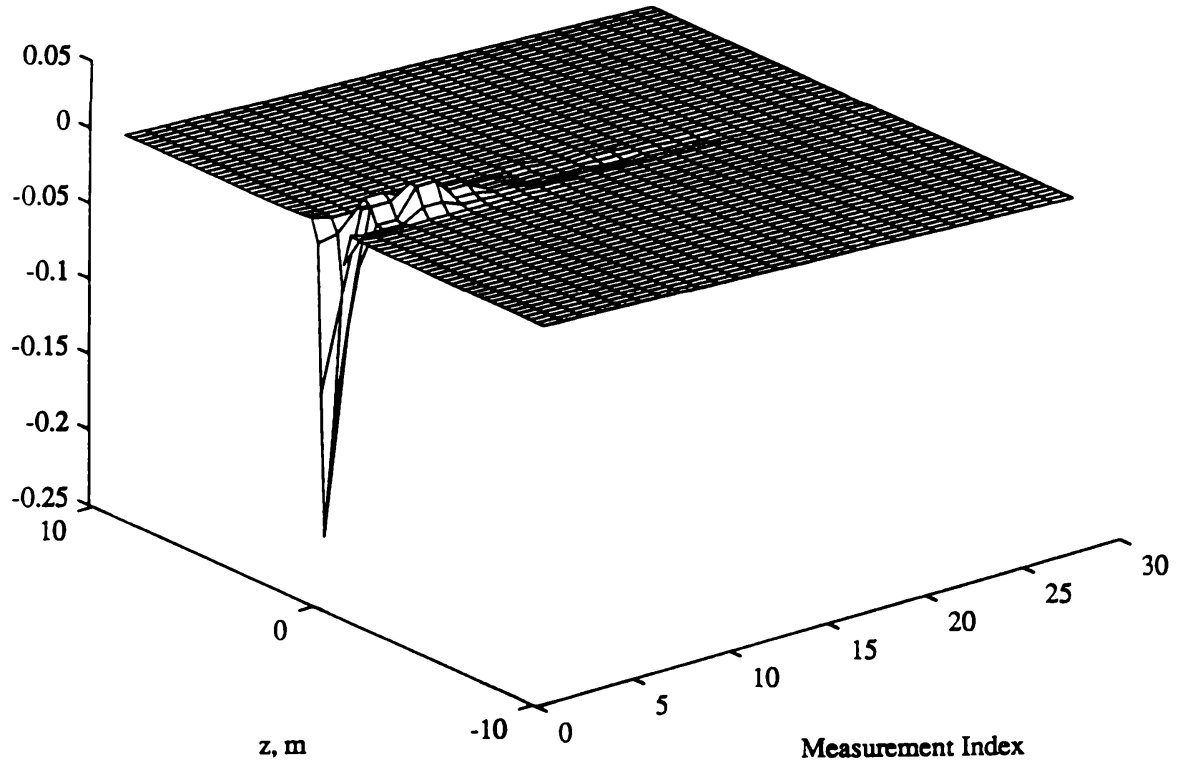

Figure 11. Deconvolution filter set for the first projection; $m$ th column corresponds to $h_{1 m}$.

application, the following form of Blackman window is used [Jackson, 1986]: $\operatorname{wndw}_{B}(k)=0.42+0.5 \cos \left(\frac{\pi}{k_{0}} k\right)+0.08 \cos \left(\frac{2 \pi}{k_{0}} k\right)$.
The required computation for (38) can be performed efficiently by using FFT techniques. The deconvolution filters corresponding to the first and last projections obtained in this way are shown in Figure 11 and 12 , respectively. In general, as it is seen (39) in these figures, the deconvolution filters $\mathbf{h}_{\text {im }}$ have

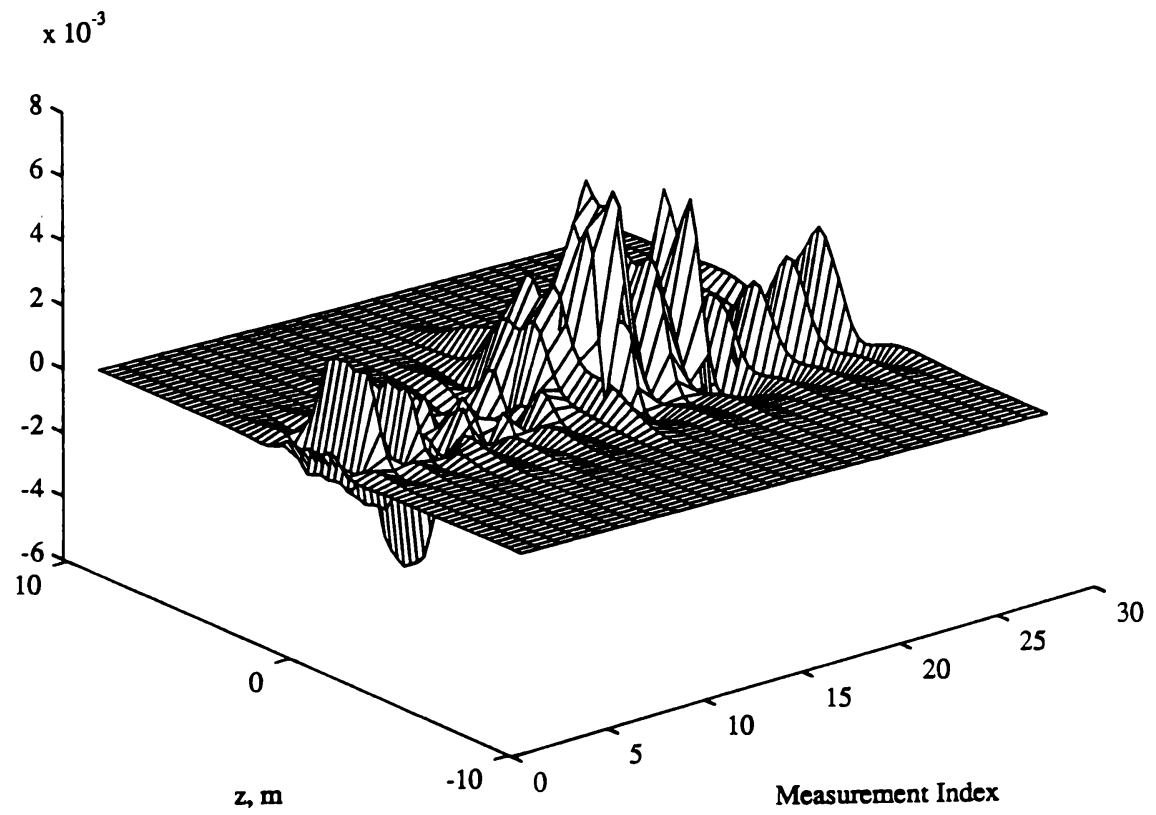

Figure 12. Deconvolution filter set for the last projection; $m$ th column corresponds to $h_{11 m}$. 


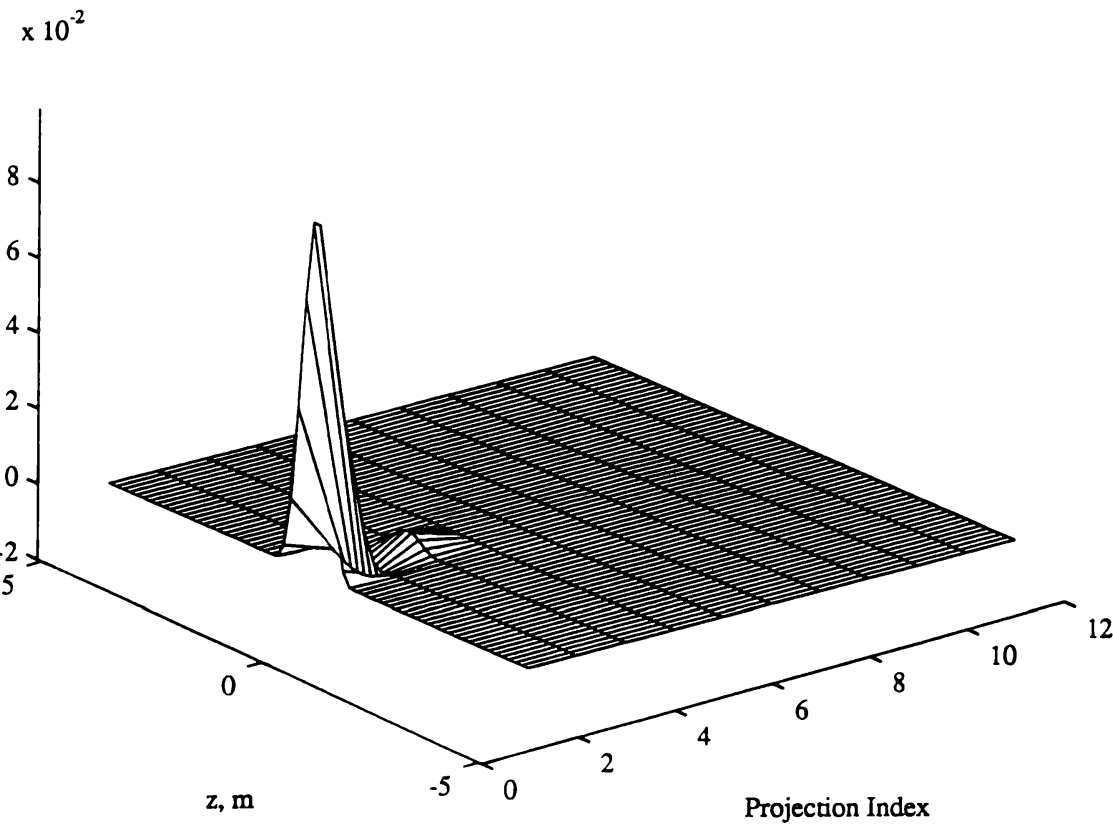

Figure 13. The parameter $h s_{1 m^{\prime}}$. Assessing performance of multichannel deconvolution result for the first projection.

lower-frequency contents for larger $i$ and $m$. One way of assessing the quality of deconvolution is finding the relationship between original and estimated projections, which can be obtained by using (10) and (11) as

$$
\begin{aligned}
& \hat{p}_{m}(z)=\sum_{i=1}^{I} \int h_{m i}\left(z-z^{\prime}\right) \\
& \cdot\left\{\sum_{m^{\prime}=1}^{M} \int s_{i m^{\prime}}\left(z^{\prime}-z^{\prime \prime}\right) p_{m^{\prime}}\left(z^{\prime \prime}\right) d z^{\prime \prime}+n_{i}\left(z^{\prime}\right)\right\} \\
& \hat{p}_{m}(z)=\sum_{m^{\prime}=1}^{M}\left(h s_{m m^{\prime}} * p_{m^{\prime}}\right)(z)+h n_{m}(z),
\end{aligned}
$$

where

$$
h s_{m m^{\prime}}(z)=\sum_{i=1}^{I} \int h_{m i}\left(z-z^{\prime}\right) s_{i m^{\prime}}\left(z^{\prime}\right) d z^{\prime}
$$

and

$$
h n_{m}(z)=\sum_{i=1}^{I} \int h_{m i}\left(z-z^{\prime}\right) n_{i}\left(z^{\prime}\right) d z^{\prime}
$$

At this point, the trade-off between higher-resolution estimation of projections in the presence of noise becomes evident one more time. Higherresolution estimation of projections requires $h s_{m m}$ to be impulsive for $m=m^{\prime}$, and zero otherwise. However, in that case the variance of $h n_{m}$, which is

$$
E\left\{\left|h n_{m}(z)\right|^{2}\right\}=\sum_{i=1}^{I} \operatorname{var}_{i} \cdot\left\|h_{m i}\right\|_{2}^{2},
$$

where $\operatorname{var}_{i}$ is the variance of the $i$ th measurement noise, becomes unacceptably large. This trade-off has been considered in the computation of the deconvolution filters as described earlier in this section. For the deconvolution filters used in this application, both $h s_{m m^{\prime}}$ and the variances as in (44) have been computed. Figures 13 and 14 show three-dimensional plots of $h s_{1 m^{\prime}}$ and $h s_{3 m^{\prime}}$, respectively. As it is seen from these two figures, in general, $h s_{m m^{\prime}}$ for larger values of $m$ have smoother features, deviating from the impulsive shape described earlier. Since the actual conductivity distribution around the wellbore is usually unknown, it is difficult to judge the performance of this regularized inversion algorithm based on only actual wellbore measurements. Therefore simulated measurements 


$$
\times 10^{-2}
$$

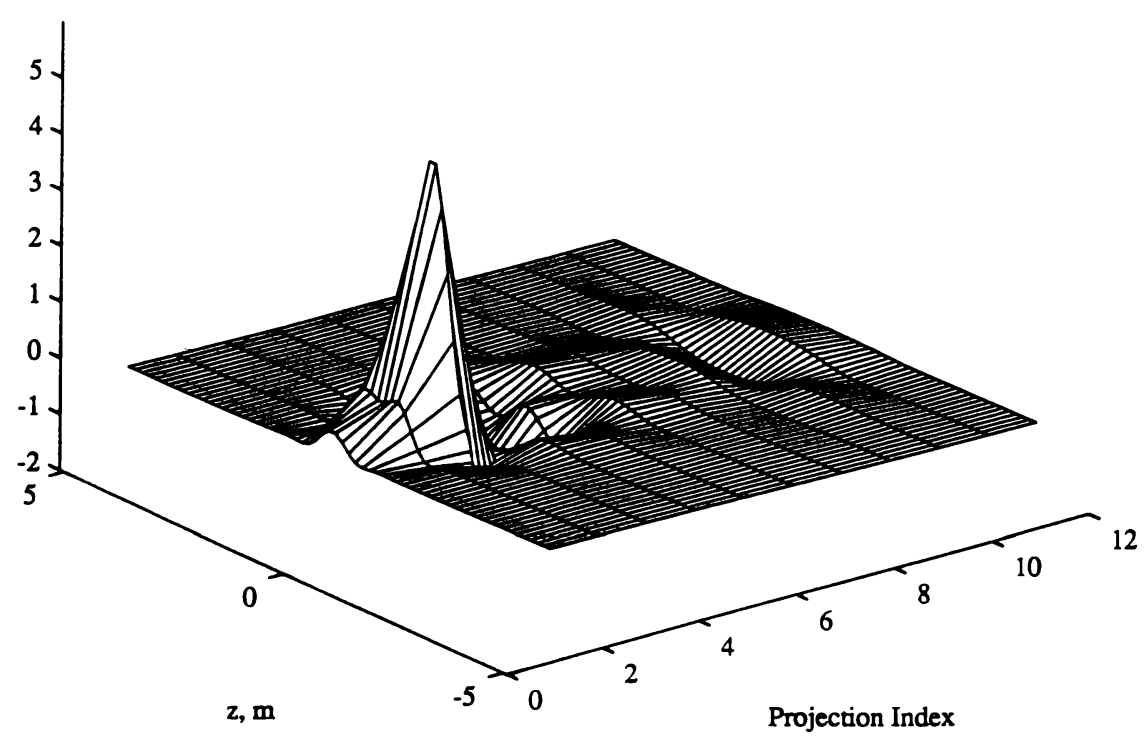

Figure 14. The parameter $h s_{3 m^{\prime}}$. Assessing performance of multichannel deconvolution result for the third projection.

of known conductivity distributions are used for this purpose. In this section the performance of the algorithm is investigated based on two reconstructions, beginning from a set of simulated and actual measurements. In Figure 15 the conductivity distribution used in the preparation of a synthetic measurement set is shown. This type of layered conductivity distributions is commonly used as a formation model in oil exploration. It is important to note that the synthetic measurement set is not obtained through the approximate measurement relation used in the inversion algorithm in (5); rather, it is provided by accurate numerical algorithms that simulate the measurements in such layered conductivity distributions [Chen et al., 1991]. The wellbore radius is set to $7.6 \mathrm{~cm}$, and the

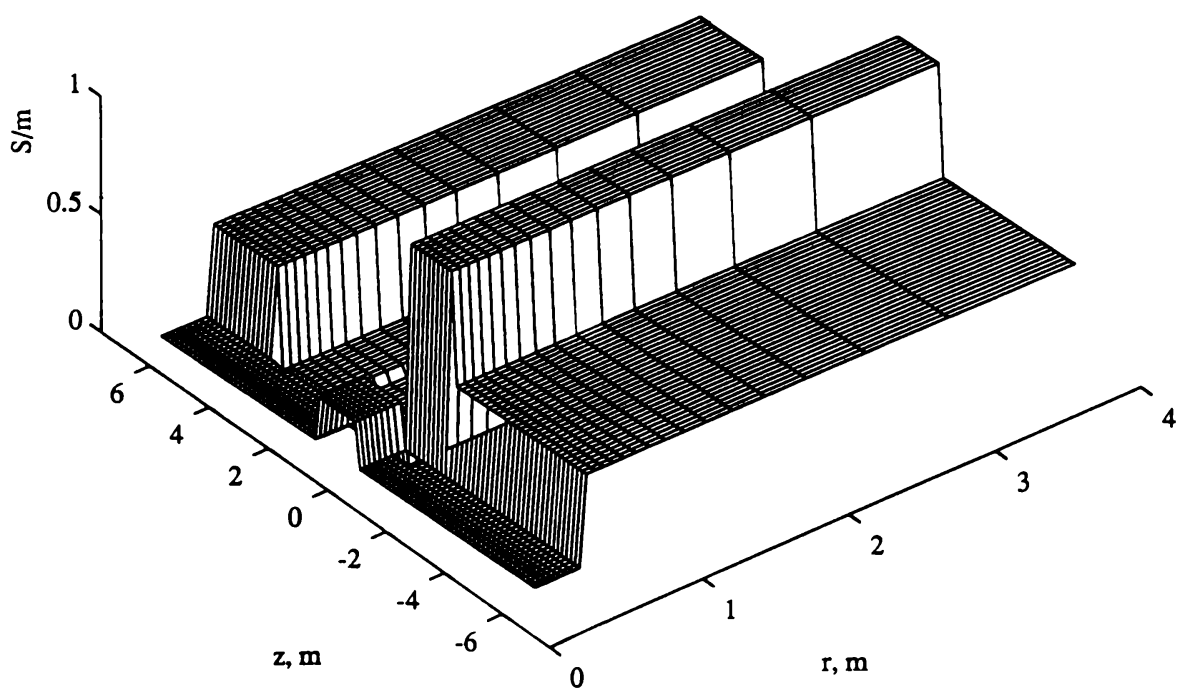

Figure 15. Actual layered conductivity distribution used in generation of synthetic measurements. 


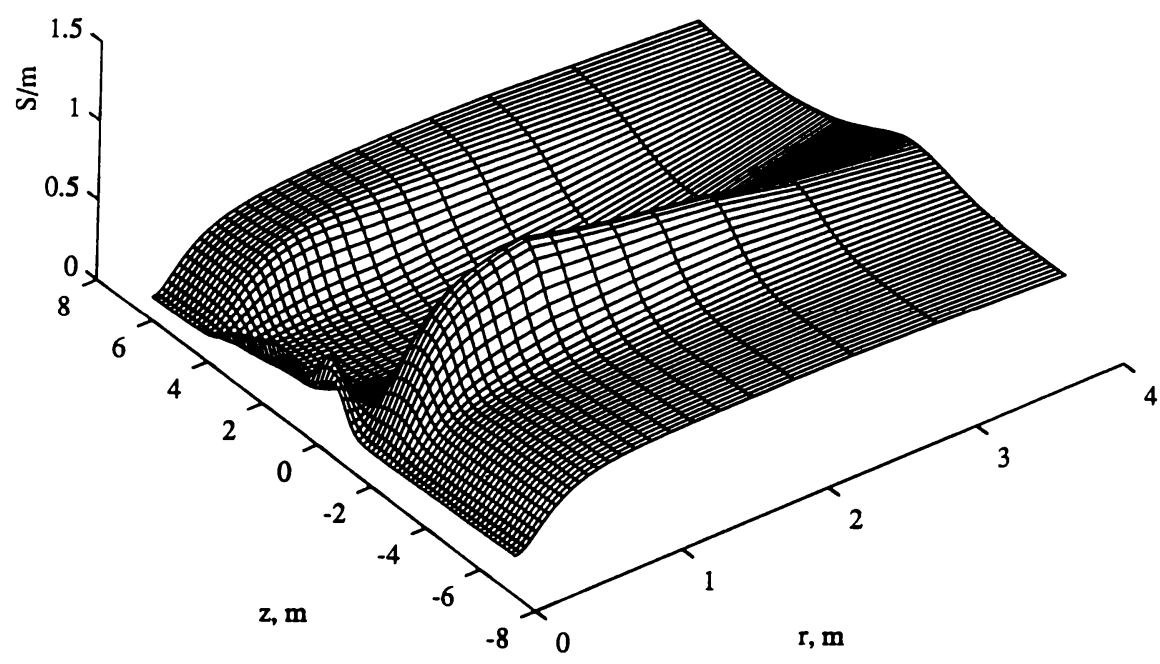

Figure 16. Reconstruction based on synthetic measurements.

conductivity of the mud inside the wellbore is chosen as $0.5 \mathrm{~S} \mathrm{~m}^{-1}$. The background conductivity $\sigma_{0}$ is also chosen as $0.5 \mathrm{~S} \mathrm{~m}^{-1}$. All of the 28 measurements are simulated at every $7.6 \mathrm{~cm}$ of $z$ increments. The reconstruction of conductivity based on these measurements is shown in Figure 16. As it is seen in this figure, reconstructions obtained by this inversion algorithm are smoother than the actual conductivity distribution. Also, estimates further away from the borehole axis are smoother than the
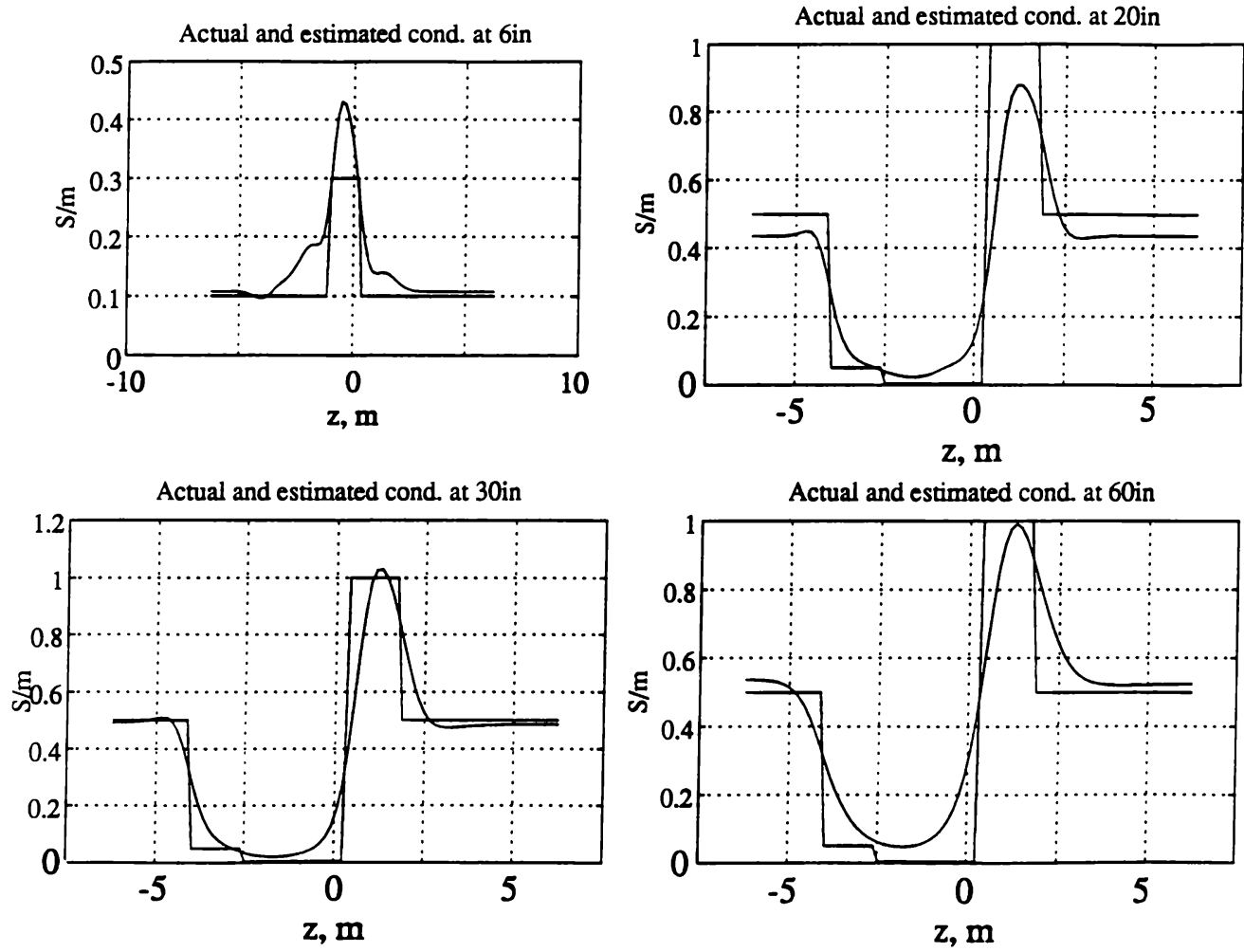

Figure 17. Four vertical slices of actual and reconstructed conductivity taken at different radial distances; actual conductivity profile is piecewise constant. 


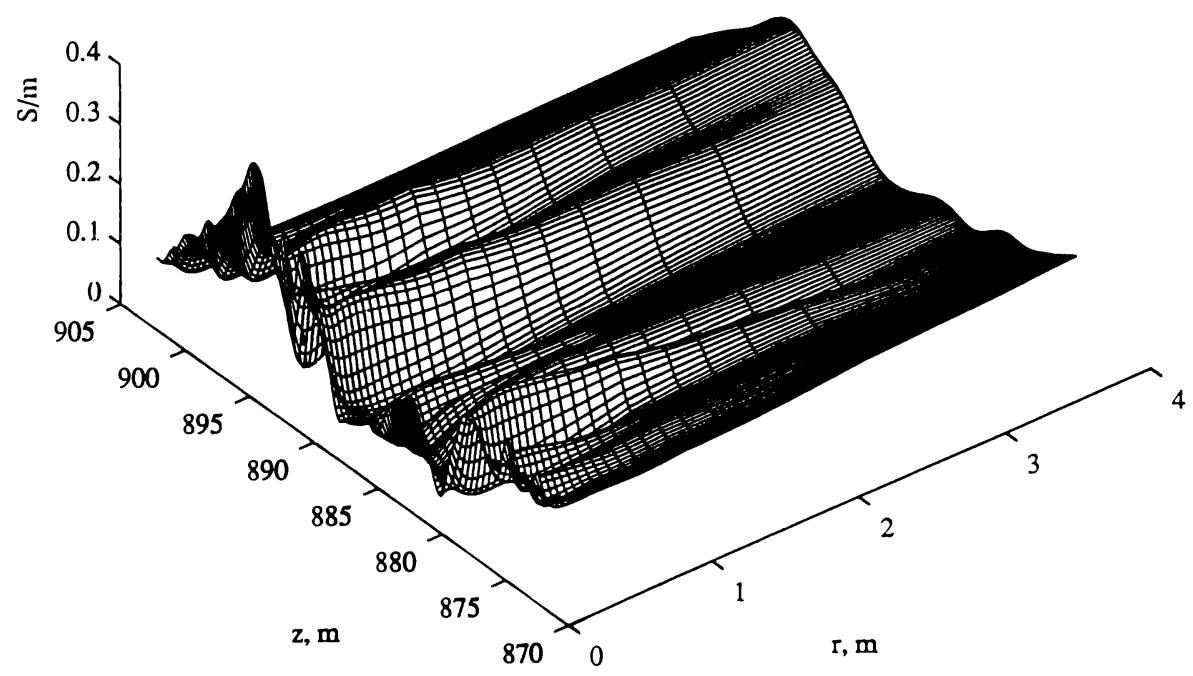

Figure 18. Reconstruction based on actual wellbore induction measurements of conductivity.

closer ones.-For further comparison, four slices of both actual and the reconstructed conductivity distributions taken at $15.2,50.8,76.2$, and $152.4 \mathrm{~cm}$ away from the borehole axis are shown in Figure 17. As it was pointed out earlier, in practice, it is important to have reliable estimates of conductivity beyond the invaded zone, which is usually $25-75 \mathrm{~cm}$ away from the borehole axis. Comparison of slices at 76.2 and $152.4 \mathrm{~cm}$ shows that the performance of the algorithm is acceptable in this respect. Also, from this plot it is seen that reconstructed conductivity distribution gradually approaches to the assumed background value beyond $230 \mathrm{~cm}$.

A reconstruction based on actual wellbore measurements is shown in Figure 18. Prior geological study of this region has revealed a layered formation with no hydrocarbon presence. During the drilling phase a relatively conductive drilling mud has been used. Therefore as it is the case in this reconstruction, it is expected to see more conductive estimates for the shallower regions. Also, the characteristic gradual decrease in resolution with radial depth is apparent in this reconstruction. This effect is more severe when the variable change $r=$ $\phi\left(r^{\prime}\right)$ is not used.

\section{Conclusions}

In this paper a regularized inversion algorithm for a two-dimensional integral equation, which is used in modeling measurements of wellbore conductivity and gravity of rock formations, and profiling mag- netotelluric surface fields, is given. Inversion algorithm involves two efficient processing steps: multichannel deconvolution of measurements in the first dimension and then backprojection onto the resolvable subspace in the second dimension. A constrained regularization in deconvolution stage is given. Some important details of the algorithm are examined in an application to wellbore induction measurements of formation conductivity.

\section{Appendix}

In this appendix the optimization problem formulated in (22) and (23) is investigated to provide an efficient iterative algorithm for its solution.

For every $\omega$ the optimization problem is in the following form: minimize

$$
\|\mathrm{HS}-1\|_{F}^{2}
$$

subject to trace

$$
\left(\mathrm{HR}_{N} \mathrm{H}^{H}\right) \leq \varepsilon^{2}
$$

where $\mathrm{R}_{N}$, autocorrelation of $N$, is positive definite and Hermitian. Through using this property of $\mathrm{R}_{N}$, the following definitions help reformulate the optimization problem in a simpler form:

$$
\begin{gathered}
\mathrm{B}=\mathrm{HR}_{N}^{1 / 2} \\
\mathrm{~A}=\mathrm{R}_{N}^{-1 / 2} \mathrm{~S}
\end{gathered}
$$


giving the following form to be minimized with respect to $B$ : minimize

$$
\|\mathrm{BA}-\|_{F}^{2}
$$

subject to trace

$$
\left(\mathrm{BB}^{H}\right) \leq \varepsilon^{2} .
$$

Minimization of (49) in the absence of any constraint is straightforward [Dennis and Schnabel, 1983]. Therefore if this solution satisfies the constraint in (50), then it is the solution to the constrained problem. Hence in the following, it is assumed that the solution to the unconstrained case does not satisfy the constraint; that is, the constraint is active.

Assuming $\mathrm{A}_{N \times M}$ with $M \leq N$, it can be expanded in its singular value decomposition as

$$
\mathrm{A}=\sum_{i=1}^{M} \lambda_{i} \mathbf{u}_{i} \mathbf{v}_{i}^{H}
$$

where $\lambda_{i} \geq 0$, and both $\mathbf{u}_{i}$ and $\mathbf{v}_{i}$ forms orthonormal sets. Since in this decomposition the case of zero singular values is not ruled out, $\mathbf{v}_{i}$ form an orthonormal basis for $\mathrm{R}^{M}$. Although $\mathbf{u}_{i}$ form an orthogonal basis for possibly a strict subspace of $\mathrm{R}^{N}$, they can be augmented by $\mathbf{u}_{i}, M+1 \leq i \leq N$, to form an orthonormal basis for $\mathrm{R}^{N}$

$\left[\mathbf{u}_{1} \cdots \mathbf{u}_{M} \mathbf{u}_{M+1} \cdots \mathbf{u}_{N}\right]\left[\mathbf{u}_{1} \cdots \mathbf{u}_{M} \mathbf{u}_{M+1} \cdots \mathbf{u}_{N}\right]^{H}=I_{N \times N}$.

In the following analysis, it is shown that these added vectors lie in the null space of the optimal B which has the following singular value decomposition:

$$
\mathrm{B}=\sum_{i=1}^{M} \gamma_{i} \mathbf{v}_{i} \mathbf{u}_{i}^{H}
$$

and an iterative minimization algorithm is given to choose optimal $\gamma_{i}$ values. There are different ways of showing that optimal SVD of B is in this form. The following is more of a direct way of proving (53). The case of $M>N$ can be analyzed in a very similar way. At the end of the following analysis, the required modifications for this case are pointed out.
Using (48) in (49) and noting that $\sum_{i=1}^{M} \mathbf{v}_{i} \mathbf{v}_{i}^{H}=\mathrm{I}$, give

$$
\|\mathrm{BA}-\|_{F}^{2}=\left\|\sum_{i=1}^{M}\left\{\lambda_{i} \mathbf{w}_{i}-\mathbf{v}_{i}\right\} \mathbf{v}_{i}^{H}\right\|_{F}^{2}
$$

where

$\mathrm{w}_{i}=\mathrm{Bu}_{i}, \quad 1 \leq i \leq N$.

The right-hand side of (54) can be simplified by the following steps:

$$
\begin{aligned}
& \left\|\sum_{i=1}^{M}\left\{\lambda_{i} \mathbf{w}_{i}-\mathbf{v}_{i}\right\} \mathbf{v}_{i}^{H}\right\|_{F}^{2} \\
& =\sum_{k=1}^{M} \sum_{l=1}^{M}\left\{\sum_{i=1}^{M} \sum_{j=1}^{M}\left(\lambda_{i} w_{i k}-v_{i k}\right) v_{i l}^{*} v_{j l}\left(\lambda_{j} w_{j k}^{*}-v_{j k}^{*}\right)\right\} \\
& =\sum_{i=1}^{M} \sum_{j=1}^{M} \sum_{k=1}^{M}\left(\lambda_{i} w_{i k}-v_{i k}\right)\left(\lambda_{j} w_{j k}^{*}-v_{j k}^{*}\right)\left\{\sum_{l=1}^{M} v_{i l}^{*} v_{j l}\right\} \\
& =\sum_{i=1}^{M} \sum_{k=1}^{M}\left|\lambda_{i} w_{i k}-v_{i k}\right|^{2} \\
& =\sum_{i=1}^{M}\left\|\lambda_{i} \mathbf{w}_{i}-\mathbf{v}_{i}\right\|^{2},
\end{aligned}
$$

in the second step, the orthogonality of $\mathbf{v}_{i}$ to $\mathbf{v}_{j}$ for $i \neq j$ is used. Since, the augmented $u_{i}$ have the property in (52), the left-hand side of the constraint in (50) can be expressed in terms of $\mathbf{w}_{i}$ as follows:

$$
\begin{aligned}
\operatorname{trace}\left(\mathrm{BB}^{H}\right)= & \operatorname{trace}\left(\left\{\mathrm{B}\left[\mathbf{u}_{1} \cdots \mathbf{u}_{M} \mathbf{u}_{M+1} \cdots \mathbf{u}_{N}\right]\right\}\right. \\
\cdot\left\{\mathrm { B } \left[\mathbf{u}_{1} \cdots\right.\right. & \left.\left.\left.\mathbf{u}_{M} \mathbf{u}_{M+1} \cdots \mathbf{u}_{N}\right]\right\}^{H}\right) \\
= & \operatorname{trace}\left(\left\{\left[\mathbf{w}_{1} \cdots \mathbf{w}_{M} \mathbf{w}_{M+1} \cdots \mathbf{w}_{N}\right]\right\}\right. \\
& \left.\cdot\left\{\left[\mathbf{w}_{1} \cdots \mathbf{w}_{M} \mathbf{w}_{M+1} \cdots \mathbf{w}_{N}\right]\right\}^{H}\right) \\
= & \sum_{i=1}^{M}\left\|\mathbf{w}_{i}\right\|^{2}+\sum_{i=M+1}^{N}\left\|\mathbf{w}_{i}\right\|^{2},
\end{aligned}
$$

leading to the following expression for the constraint in (50) in terms of $\mathbf{w}_{i}$ : 


$$
\sum_{i=1}^{M}\left\|\mathbf{w}_{i}\right\|^{2}+\sum_{i=M+1}^{N}\left\|\mathbf{w}_{i}\right\|^{2} \leq \varepsilon^{2}
$$

From this form of the constraint and the fact that the cost to be minimized depends only on $w_{i}$ for $1 \leq$ $i \leq M$, it can be concluded that for the active constraint case, the solution to the following optimization problem is the same as the original problem: minimize

$$
\sum_{i=1}^{M}\left\|\lambda_{i} \mathbf{w}_{i}-\mathbf{v}_{i}\right\|^{2}
$$

subject to

$$
\sum_{i=1}^{M}\left\|w_{i}\right\|^{2} \leq \varepsilon^{2}
$$

This implies that the optimal $\mathrm{B}$ has $\mathbf{w}_{i}=0$ for $M<$ $i \leq N$ and it can be expressed as

$$
\mathrm{B}^{*}=\sum_{i=1}^{M} \mathbf{w}_{i} \mathbf{u}_{i}^{H}
$$

The optimization problem of (60) and (61) can be solved by using method of Lagrange multiplier [Vogel et al., 1990], providing

$$
\mathbf{w}_{i}^{*}=\frac{\lambda_{i}}{\lambda_{i}^{2}+\mu^{2}} \mathbf{v}_{i}
$$

where $\mu \geq 0$ is the unique solution to

$$
\sum_{i=1}^{M}\left(\frac{\lambda_{i}}{\lambda_{i}^{2}+\mu^{2}}\right)^{2}=\varepsilon^{2}
$$

The active constraint assumption guarantees the existence of a solution for this equation. Since the equation involves powers of $\mu^{2}$, when $\mu_{0}$ solves it so does $-\mu_{0}$. The fact that the left-hand side of (64) is a monotonic decreasing function of $\mu>0$, with the derivative

$$
\sum_{i=1}^{M}-\frac{4 \mu \lambda_{i}^{2}}{\left(\lambda_{i}^{2}+\mu^{2}\right)^{3}}<0
$$

implies that there is only one positive $\mu$ that solves (64). An iterative algorithm such as the Gauss-
Newton algorithm can be used to find this unique positive solution, and the derivative given in (65) can be used in this process.

After finding $\mu$ that solves (64), optimal B* can be found by using (63) and (62) as

$$
\mathrm{B}^{*}=\sum_{i=1}^{M} \frac{\lambda_{i}}{\lambda_{i}^{2}+\mu^{2}} \mathbf{v}_{i} \mathbf{u}_{i}^{H}
$$

which is actually SVD of B* and it is in the form given in (53). Then, the optimal solution to the original optimization problem can be found by using (47) as

$$
\mathrm{H}^{*}=\mathrm{B}^{*} \mathrm{R}_{N}^{-1 / 2}
$$

The optimal solution $\mathrm{H}^{*}$ can be rewritten in the following form in terms of the original matrices by using (48) and (51) as

$$
\mathrm{H}^{*}=\left(\mathrm{S}^{H} \mathrm{R}_{N}^{-1} \mathrm{~S}+\mu^{2} \mathrm{I}\right)^{-1} \mathrm{~S}^{H} \mathrm{R}_{N}^{-1} .
$$

In the above derivation, the case of $M \leq N$ has been assumed. When $M>N$, with minor modifications, the above approach can be used to prove that

$$
\mathrm{B}^{*}=\sum_{i=1}^{N} \frac{\lambda_{i}}{\lambda_{i}^{2}+\mu^{2}} \mathbf{v}_{i} \mathbf{u}_{i}^{H}
$$

where $\mu$ solves

$$
\sum_{i=1}^{N}\left(\frac{\lambda_{i}}{\lambda_{i}^{2}+\mu^{2}}\right)^{2}=\varepsilon^{2} .
$$

As before, the optimal solution to the original optimization problem can be found by using (67). In this case, in (51), there are $N$ terms with leftsingular vectors $\mathbf{u}_{i}$ forming an orthonormal basis for $\mathrm{R}^{N}$. However, $\mathrm{v}_{i}$ have to be augmented to form an orthonormal basis for $\mathrm{R}^{M}$. Then, the minimization problem in terms of $\mathbf{w}_{i}$, as defined in (60) and (61) follows: minimize

$$
\sum_{i=1}^{N}\left\|\lambda_{i} \mathbf{w}_{i}-\mathbf{v}_{i}\right\|^{2}+\sum_{i=N+1}^{M}\left\|\mathbf{v}_{i}\right\|^{2}
$$

subject to 


$$
\sum_{i=1}^{N}\left\|\mathbf{w}_{i}\right\|^{2} \leq \varepsilon^{2}
$$

Since the second summation in the cost expression is independent of $\mathbf{w}_{i}$, it can equivalently be rewritten as the following: minimize

$$
\sum_{i=1}^{N}\left\|\lambda_{i} \mathbf{w}_{i}-\mathbf{v}_{i}\right\|^{2}
$$

subject to

$$
\sum_{i=1}^{N}\left\|w_{i}\right\|^{2} \leq \varepsilon^{2}
$$

which can be solved as described earlier for the case of $M \leq N$ to obtain optimal solutions as in (69), (70), and (47).

Acknowledgments. I would like to thank my colleagues and friends Tarek M. Habashy, Dave J. Rossi, Apo Sezginer and Carlos Torres-Verdin of Schlumberger-Doll Research for their helpful ideas at many stages of this work. I also want to thank Alan S. Willsky of MIT for his insightful comments and suggestions, and Qing-Huo Liu for his help in generating the synthetic measurement set used in one of the examples. Finally, I wish to thank the anonymous reviewers of the manuscript.

\section{References}

Barber, T. D., and Rosthal, R. A., Using a multiarray induction to achieve high-resolution logs with minimum environmental effects, Proc. of 66th Ann. Tech. Conf. Exhib. Soc. Pet. Eng., SPE-22725, 637-651, 1991.

Chambers, L. G., Integral Equations: A Short Course, International Textbooks, Scranton, Pa., 1976.

Chew, W. C., Z. Nie, Q.-H. Liu, and B. Anderson, An efficient solution for the response of electrical well logging tools in a complex environment, IEEE Trans. Geosci. Remote Sens., 29, 308-313, 1991.

Delves, L. M., and J. L. Mohamed, Computational Methods for Integral Equations, Cambridge University Press, Cambridge, 1985.

Dennis, J. E., and R. B. Schnabel, Numerical Methods for Unconstrained Optimization and Nonlinear Equations, Prentice-Hall, Englewood Cliffs, N. J., 1983.

Dunford, N., and J. T. Schwartz, Linear Operators, Wiley Interscience, New York, 1964.

Ellis, D. V., Well Logging for Earth Scientists, Elsevier, New York, 1987.
Golub, G. H., and C. F. Van Loan, Matrix Computations, 2nd ed., Baltimore: John Hopkins University Press, Baltimore, 1989.

Habashy, T. M., and R. Mittra, On some inverse methods in electromagnetics, J. Electromagn. Waves Appl., 1, 25-58, 1987.

Habashy, T. M., R. W. Groom, and B. R. Spies, Beyond the Born and Rytov approximations: A nonlinear approach to electromagnetic scattering, 98, 1759-1775, 1993.

Hanson, R. J., A numerical method for solving Fredholm integral equations of the first kind using singular values, SIAM J. Num. Anal., 8, 616-622, 1971.

Hunka, J. F., et al., A new resistivity measurement system for deep formation imaging and high-resolution formation evaluation, Proc. of 65th Ann. Tech. Conf. Exhib. Soc. Petr. Eng., SPE-20559, pp. 295-307, New Orleans, LA, Sept. 23-26, 1990.

Jackson, L. B., Digital Filters and Signal Processing, Kluwer Academic, Norwell, Mass., 1986.

Keener, J. P., Principles of Applied Mathematics, Addison-Wesley, New York, 1988.

Moran, J . H., and K. S. Kunz, Basic theory of induction logging and application to study of two coil sondes, Geophysics, 27, 829-858, 1962.

Pipkin, A. C., A Course on Integral Equations, SpringerVerlag, New York, 1991.

Sezginer, A., The inverse source problems of magnetostatics and electrostatics, Inverse Problems, 3, L87L91, 1987.

Smithies, F., Integral Equations, Cambridge University Press, London, 1958.

Tikhonov, A. N., and Arsenin, V. Y., Solutions of Ill-Posed Problems, John Wiley, New York, 1977.

Torres-Verdin, C., H. F. Morrison, and F. X. Bostick, Jr., Born inversion of magnetotelluric data, paper presented at IAGA 10th International Workshop on EM Induction in the Earth and Moon, Int. Assoc. of Geomagn. and Aeron., Rockville, Md., Aug. 22-29, 1990.

Van Trees, H. L., Detection, Estimation, and Modulation Theory, Part 1, John Wiley, New York, 1968.

Vogel, C. R., A constrained least squares regularization method for nonlinear ill-posed problems, SIAM J. Control Optim., 28, 34-49, 1990.

Yaglom, A. M., An Introduction to the Theory of Stationary Random Functions, Dover, New York, 1962.

O. Arıkan, Electrical Engineering Department, Bilkent University, Bilkent, Ankara 06533, Turkey.

(Received October 8, 1992; revised May 19, 1993; accepted June 8, 1993.) 